\title{
Three new species and two new records of the genus Cotesia Cameron (Hymenoptera: Braconidae) from Iran
}

\author{
Mohammad ZARGAR ${ }^{1}$, Ankita GUPTA ${ }^{2}$, \\ Ali Asghar TALEBI ${ }^{3,{ }^{*}}$ \& Samira FARAHANI ${ }^{4}$ \\ ${ }^{1,3}$ Department of Entomology, Faculty of Agriculture, Tarbiat Modares University, \\ P.O. Box 14115-336, Tehran, Iran. \\ ${ }^{2}$ ICAR-National Bureau of Agricultural Insects Resources, P.B. No. 2491, \\ H.A. Farm Post, Bellary Road, Hebbal, 560024 Bangalore, India. \\ ${ }^{4}$ Research Institute of Forests and Rangelands, Agricultural Research Education and Extension \\ Organization (AREEO), P.O. Box 13185-116, Tehran, Iran. \\ *Corresponding author: talebia@modares.ac.ir \\ ${ }^{1}$ Email: mohammad.zargar@modares.ac.ir \\ 2Email: drankitagupta7@gmail.com \\ ${ }^{4}$ Email: s.farahani@rifr-ac.ir

\footnotetext{
${ }^{1}$ urn:1sid:zoobank.org:author:6F685437-6655-4D8B-9DD5-C66A0824B987

${ }^{2}$ urn:1sid:zoobank.org:author:AC7B7E50-D525-4630-B1E9-365ED5511B79

${ }^{3}$ urn:1sid:zoobank.org:author:71CB13A9-F9BD-4DDE-8CB1-A495036975FE

${ }^{4}$ urn:1sid:zoobank.org:author:423DEB84-81C3-4179-BDE2-88A827CD4865
}

\begin{abstract}
The present study is based on the genus Cotesia Cameron,1891 collected from Khuzestan Province in the Southwestern part of Iran during 2016-2017. Nine species (+200 specimens) of the genus Cotesia were collected and identified. We recognised three new species, which we describe and illustrate here: Cotesia elongata Zargar \& Gupta sp. nov., C. khuzestanesis Zargar \& Gupta sp. nov. and C. zagrosensis Zargar \& Gupta sp. nov. Two species are recorded for the first time from Iran: Cotesia cynthiae (Nixon, 1974) and C. glabrata (Telenga, 1955). A faunistic list with species distribution in Iran, a modified key to include the new species and brief diagnoses for the new records from Iran are provided.
\end{abstract}

Keywords. Cotesia, Khuzestan, faunistic list, modified key.

Zargar M., Gupta A., Talebi A.A. \& Farahani S. 2019. Three new species and two new records of the genus Cotesia Cameron (Hymenoptera: Braconidae) from Iran. European Journal of Taxonomy 571: 1-25.

https://doi.org/10.5852/ejt.2019.571

\section{Introduction}

Braconidae Nees, 1811 is the second largest family of Hymenoptera Linnaeus, 1758, and the subfamily Microgastrinae Förster, 1862 is known with over 2710 described species worldwide (Yu et al. 2016). 
Microgastrinae is one of the largest groups of parasitoids in terms of both species richness and economic importance (Rodriguez et al. 2013). The genus Cotesia Cameron, 1891 (Hymenoptera: Braconidae), with 296 described species worldwide, is one of the largest genera of parasitoid wasps in the megadiverse subfamily Microgastrinae (Yu et al. 2016). The actual diversity of Cotesia has been estimated from 1500 (Mason 1981) to 2500 species around the world (van Achterberg \& Polaszek 1996). The genus Cotesia was erected by Cameron (1891), but was later synonymized with Apanteles Szépligeti, 1904 (Szépligeti 1904) until the generic reclassification of the Microgastrinae by Mason (1981).

Microgastrine genera are either solitary or gregarious endoparasitoids. Small broods with two or three individuals are known in a few cases, e.g., C. astrarches (Marshall, 1889) with 3-6 indiviuals in the host Aricia Reichenbach, 1817 (Lepidoptera Linnaeus, 1758: Lycaenidae Leach, 1815) (Shaw 2012; Quicke 2015). Gupta et al. (2016a) used the gregarious species of the genus Glyptapanteles Ashmead, 1904 to generate accurate boundaries between species/species-groups using an integrated approach with three different sets of data (morphology, host records and mitochondrial cytochrome c oxidase subunit I(COI) nucleotide sequences). Gupta \& Fernández-Triana (2014) and Fernández-Triana et al. (2014) recorded many gregarious and solitary hosts associated with Cotesia from the Oriental and Neotropical regions, respecively. Species of this genus are selected regularly as biological control agents. Cotesia flavipes Cameron, 1891 and other closely allied species, such as C. chilonis (Munakata, 1912), C. sesamiae (Cameron, 1906) and C. nonagriae (Olliff, 1893), attack a wide range of sugarcane pests, such as Chilo partellus (Swinhoe, 1885) and C. sacchariphagus (Bojer, 1856) (Lepidoptera: Crambidae Latreille, 1810) (Quicke 2015). Several species of Cotesia have also been applied as model organisms in physiology, ecology and population genetics studies (Michel-Salzat \& Whitfield 2004).

Despite the immense species diversity of the genus, the members tend to appear relatively uniform morphologically. Cotesia can be identified by the shape of the first and second tergites and propodeum sculpture. The first tergite never narrows apically, is a little longer than wide and broadens apically, but sometimes is wider than long; the width of the second tergite is 1.5 times the apical width of the first tergite or less. The propodeum is rugose and never has an areola, usually with a median longitudinal carina that sometimes becomes partly obscured by rugosity. Until now, only two species, Cotesia pistrinariae (Wilkinson, 1929) and Cotesia trabalae Gupta, 2016, across the globe are known to have a strongly narrowing first tergite at midlength (Gupta et al. 2016b).

The faunal studies on the subfamily Microgastrinae are gaining importance in Iran (Farahani et al. 2014, 2016; Gadallah et al. 2015; Ghafouri Moghaddam et al. 2018; Abdoli et al. 2019a, 2019b; Zargar et al. 2019a, 2019b). To date, 34 species of the genus Cotesia have been reported from Iran (Farahani et al. 2016; Samin et al. 2018). Considering the species richness and poor number of known species from Iran, detailed taxonomic and faunistic studies on this subfamily are essentially warranted. In the present study on the Microgastrinae fauna of the Khuzestan Province, three new species and two new distributional records are presented along with an updated faunistic list from Iran.

\section{Material and methods}

The specimens were collected from different areas of the Khuzestan Province during 2016-2017 using Malaise traps. Khuzestan Province includes mountainous areas located in the north, with plains and sea-level areas in the south. The vegetation of Khuzestan is diverse from oak forests dominated by Quercus brantii Lindley, 1840 (Fagales Engler, 1892: Fagaceae Dumort, 1829) in the highlands to marshy lands in the low elevation areas. The specimens were fortnightly removed from the collecting bottle and preserved in $70 \%$ alcohol. For mounting on card, the wasps were transferred into $70 \%, 90 \%$, and $100 \%$ alcohol, 20 minutes each for dehydration, respectively. Later, the wasps were placed on the filter paper for drying and finally were card mounted and eventually labelled. Further, the specimens were incubated in $50 \pm 5^{\circ} \mathrm{C}$ for two hours for complete dehydration. The specimens used in the present 
study are deposited in the collections of TMUC or ICAR-NBAIR. Photos of the species were taken with a Leica M 205 A stereo microscope with Leica DC 420 inbuilt camera using automontage software (ver. 3.8). Terminology and measurement follows Nixon (1965) and Mason (1981), vein terminology follows van Achterberg (1993). Nixon (1974) and Papp $(1986,1987)$ were consulted for identification.

\section{Abbreviations:}

$\mathrm{FLn}_{\mathrm{l} / \mathrm{w}} \quad=$ ratio of length of flagellomere segment to width

MOD $=$ median ocellar diameter

OOL $\quad=$ ocullar-ocellar line

$\mathrm{POL}=$ postocellar line

TI, TII, TIII = first, second and third tergite, respectively

\section{Repositories:}

ICAR-NBAIR = National Insect Museum, ICAR-National Bureau of Agricultural Insect Resources, Bengaluru, India

TMUC $=$ Department of Entomology, Tarbiat Modares University, Tehran, Iran

\section{Results}

\section{New species}

Class Insecta Linnaeus, 1758

Order Hymenoptera Linnaeus, 1758

Superfamily Ichneumonoidea Latreille, 1802

Family Braconidae Nees, 1811

Subfamily Microgastrinae Förster, 1862

Tribe Cotesiini Mason, 1981

Cotesia Cameron, 1891

Cotesia Cameron, 1891: 182-194 (type species: Cotesia flavipes (Cameron, 1891)).

Cotesia elongata Zargar \& Gupta sp. nov.

urn:lsid:zoobank.org:act:79D3997B-4118-42F3-A579-2E1B75BB4FE7

Fig. 1

\section{Diagnosis}

Penultimate segment of antenna $2.2 \times$ as long as wide; mesoscutum densely evenly punctate, in lateral lobes presence of smooth area near tegula; notauli indicated by dense punctations; scutellum sparsely punctate in anterior half, punctation dense in posterior half (Fig. 1C); pterostigma $4.0 \times$ as long as wide, light brown; vein $1-\mathrm{R} 11.4 \times$ as long as pterostigma, $3.0 \times$ as long as distance from end of vein $1-\mathrm{R} 1$ to tip of radial cell (Fig. 1D); third tergite median length $0.7 \times$ as long as second tergite (Fig. 1E).

\section{Etymology}

The name is derived from the Latin 'elongata', meaning 'elongate', and referring to the second metasomal tergite, which is longer than third tergite, while in the closely related species, Cotesia ruficrus (Haliday, 1834), it is shorter than the third tergite. 


\section{Material examined}

Holotype

IRAN - + ; Khuzestan Province, Dezful, Shahrak-e Shahid Mohammad Montazeri; 32²6'83.16" N, 48³7'67.79" E; 97 m a.s.1.; 22 May 2017; M. Zargar leg.; Malaise trap; citrus orchards; ICAR-NBAIR/ NIM/MICROG/COT/22517H.

\section{Paratypes}

IRAN • 4 O ; Khuzestan Province, Dezful, Qaleh-ye Rob-e Bandbal; 32¹7'27.94" N, 48 25'46.98" E; 97 m a.s.1.; 20 Mar.-3 Apr. 2016, 7-21 Jun. 2017; TMUC-HBMC0001-0004 - 3 o of ; Shamsabad; $32^{\circ} 29^{\prime} 64.65^{\prime \prime} \mathrm{N}, 48^{\circ} 42^{\prime} 57.45^{\prime \prime} \mathrm{E}$; 94 m a.s.1.; 8-22 May 2017, 5-19 Jun. 2017; TMUC-HBMC0005-0007• 9 우; Zoviyeh; $31^{\circ} 46^{\prime} 20.56^{\prime \prime} \mathrm{N}, 48^{\circ} 48^{\prime} 01.17^{\prime \prime}$ E; 30 m a.s.1.; 5-19 Jun. 2017; TMUC-HBMC0008-0016•

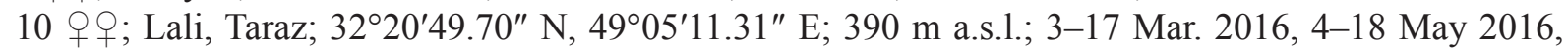
5-19 Mar. 2017, 5-19 May 2017, 22 Jun.-6 Jul. 2017; TMUC-HBMC00017-0026, ICAR-NBAIR/NIM/

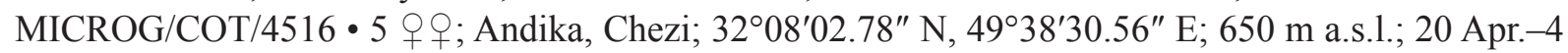
May 2016, 4-18 Jun. 2016, 22 May-6 Jun. 2017, 22 Jun.-6 Jul. 2017; TMUC-HBMC00027-0031 •

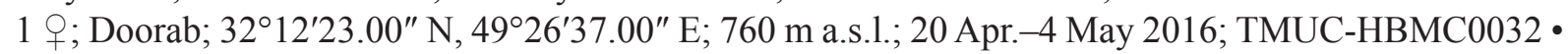
1 + ; same collection data as for preceding; 4-18 Jun. 2016; ICAR-NBAIR/NIM/MICROG/COT/4616 • 1 q; same collection data as for preceding; 4-18 Apr. 2017; NBAIR/NIM/MICROG/COT/4417 • 1 \%; same collection data as for preceding; 5-19 Jun 2017; NBAIR/NIM/MICROG/COT/5617 • 1 क; same collection data as for preceding; 22 Jun.-6 Jul. 2017; NBAIR/NIM/MICROG/COT/22617 • 6 q ; Baghmalek, Ghaletol; 31 37'49.70" N, 4952'53.35" E; 880 m a.s.1.; 3-17 Apr. 2016, 4-18 May 2016, 21 May-5 Jun. 2016, 22 Jun.-6 Jul. 2017; TMUC-HBMC0033-0038 • 1 ; ; same collection data as for preceding; 21 May-5 Jun. 2016; ICAR-NBAIR/NIM/MICROG/COT/21516 - 4 q ; Shang; 31³1'46.00" N, 4953'14.61" E; 716 m a.s.l.; 20 Mar.-4 Apr. 2016, 4-18 May 2016, 5-19 May 2017, 4-18 Jul. 2017; TMUC-HBMC0039-0042 • 2 우; Dobagh; 3131'16.14" N, 4952'53.00" E; 688 m a.s.1.; 3-17 Apr. 2016, 4-18 Jun. 2016; Malaise trap; citrus orchards; M. Zargar leg.; TMUCHBMC0043-0044 • 1 क; same collection data as for preceding; 3-17 Apr. 2016; ICAR-NBAIR/NIM/ MICROG/COT/3416.

\section{Description}

\section{Female}

MEASUREMENTs. Body length $3 \mathrm{~mm}$, fore wing length $2.9 \mathrm{~mm}$.

HEAD (Fig. 1A-B). Smooth except shallowly punctate on face; width of head in dorsal view $1.8 \times$ as long as height; width of face $1.4 \times$ as long as height; POL:MOD:OOL $4.1: 2: 4.5$; gena $0.7 \times$ as long as width of eyes; malar space $1.5 \times$ as long as width of mandibular base; antenna as long as body; flagellomeres finely setose. $\mathrm{FL}_{1 / \mathrm{w}}: 3.5, \mathrm{FL} 12_{1 / \mathrm{w}}: 2.4, \mathrm{FL}_{1 / \mathrm{w}}: 2.3, \mathrm{FL}_{1 / 1 / \mathrm{w}}: 2.2, \mathrm{FL}_{1 / \mathrm{w}}: 2.2$.

Mesosoma (Fig. 1C). Mesoscutum densely evenly punctate, in lateral lobes presence of smooth area near tegula; notauli indicated by dense punctation; scutellum weakly punctate in anterior half, densely punctate in posterior half; scutellar sulcus crenulate; postscutellum crenulate and shiny; prepectal carina absent; propodeum coarsely rugose to scabrous.

Wings (Fig. 1D). Areolet absent, vein $r$ arising little after middle of pterostigma; vein $1-R 11.4 \times$ as long as pterostigma, $2.8 \times$ as long as distance from end of vein $1-\mathrm{R} 1$ to tip of radial cell, $4.0 \times$ as long as vein r; pterostigma $4.0 \times$ as long as wide; vein $r$ as long as $2-\mathrm{SR}$; vein $1-\mathrm{cu} 1$ as long as vein 2-cu1; width of discoidal cell $1.1 \times$ as long as height.

Legs (Fig. 1F). Metacoxa $1.3 \times$ as long as first tergite; metafemur length $4.0 \times$ as long as median width; inner and outer spur of metatibia equal, $0.4 \times$ as long as basitarsus. 

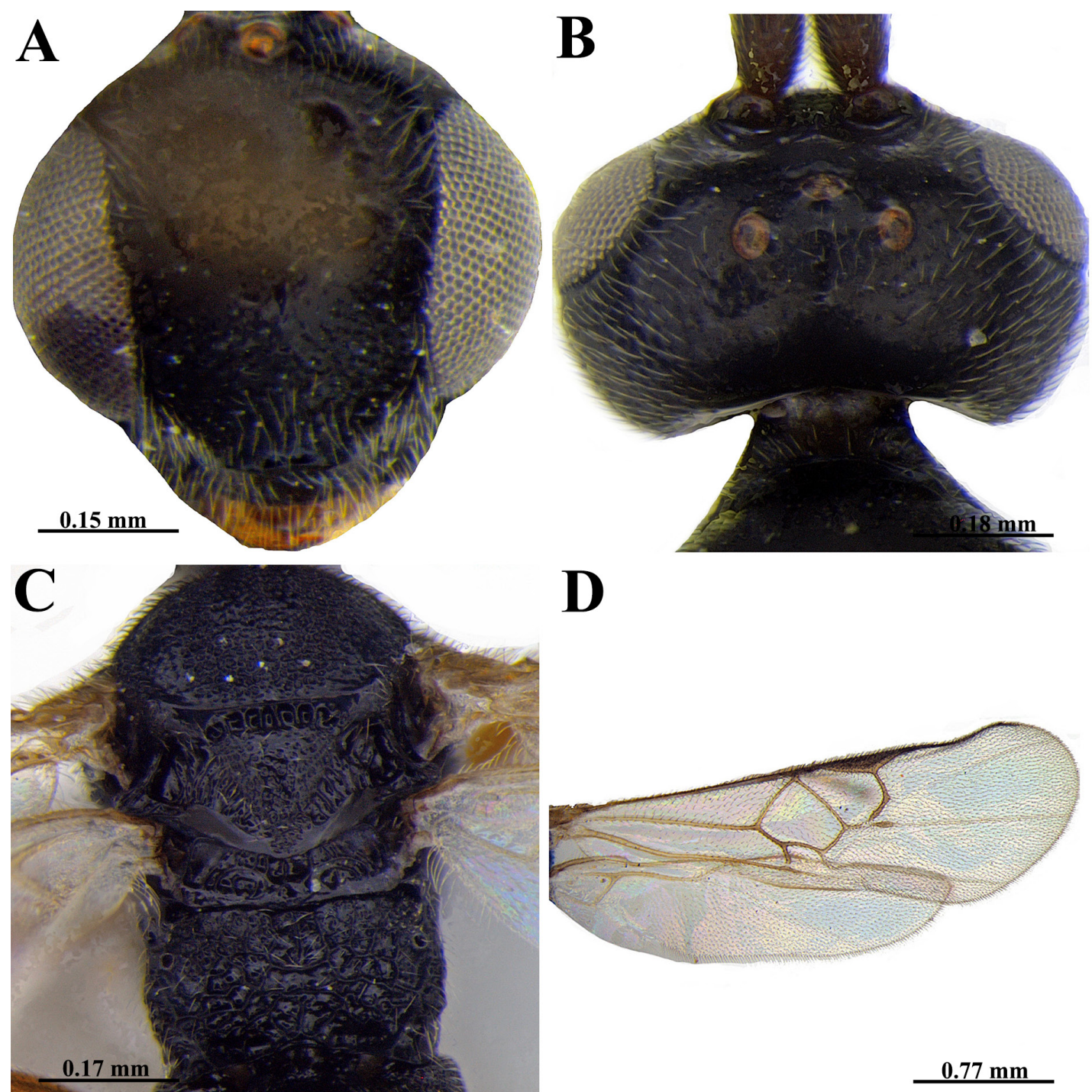

D
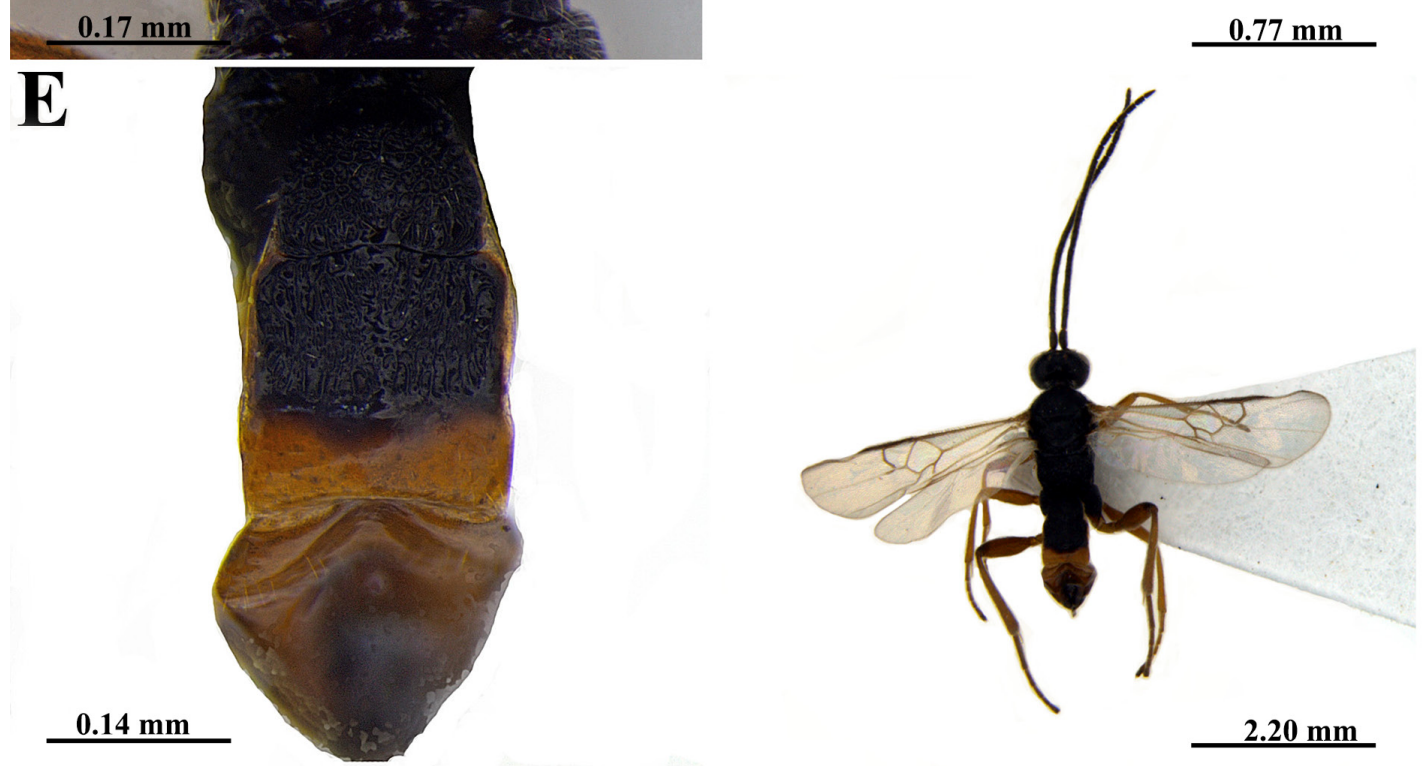

Fig. 1. Cotesia elongata Zargar \& Gupta sp. nov., ICAR-NBAIR/NIM/MICROG/COT/22517H. A. Head, frontal view. B. Head, dorsal view. C. Mesosoma, dorsal view. D. Fore wing. E. Metasoma, dorsal view. F. Habitus, dorsal view. 
Metasoma (Fig. 1E). TI slightly widening toward apex, $1.1 \times$ longer than apical width; apical width $1.7 \times$ as long as basal width, rugose. TII apical width $1.9 \times$ longer than median length. TIII median length $0.7 \times$ as long as TII; tergites posterior to TII smooth and shiny with single row of setae near hind margin; ovipositor sheath $0.5 \times$ as long as metabasitarsus; hypopygium $0.7 \times$ as long as metatibia, truncated apically.

Colour. Antenna, head, mesosoma black; metasoma yellowish brown except first and second tergite; labial palp and maxillary palp testaceous; tegula yellowish brown; profemur, mesofemur reddish brown; metafemur reddish brown except black spot at apex; tibiae reddish brown; protarsus and mesotarsus reddish brown, metatarsus dark brown; pterostigma light brown.

\section{Male \\ Unknown.}

\section{Host}

Unknown.

\section{Biology}

Unknown.

\section{Comment}

In the keys provided by Nixon (1974), Papp (1986) and van Achterberg \& Polaszek (1996), Cotesia elongata sp. nov. runs close to Cotesia ruficrus (Haliday, 1834); it can, however, be separated from the latter by the following characters: (1) penultimate segment of antenna 2-2.2 $(-2.3) \times$ as long as wide vs 1.5 and $1.7 \times$ in Nixon (1974) and van Achterberg \& Polaszek (1996), respectively, (2) third tergite $0.7-0.8 \times$ as long as second tergite (Fig. 1E) vs $1.2-1.4$ in Papp (1986).

The new species can be included in the identification key of Cotesia compiled by Nixon (1974), which is modified below:

14. Antenna short, rather thick, preapical segment about one-and-a-quarter $\times$ as long as wide; legs dark brownish or obscurely brownish yellow throughout; mesopleuron anterior to and ventral to sternaulus, very shiny and with sharp discrete punctation

C. amabilis (Nixon, 1974)

- Antenna longer and decidedly thin

A. Preapical segment of antenna fully one-and-a-half $\times$ as long as wide; mesopleuron anterior to and ventral to sternaulus, tending to be dull and evenly rugose. .C. ruficrus (Haliday, 1834)

- Preapical segment of antenna 2-2.2 $(-2.3) \times$ as long as wide; mesopleuron anterior to and ventral to sternaulus, tending to be punctate C. elongata Zargar \& Gupta sp. nov.

The new species can be included in the identification key of Cotesia compiled by Papp (1974), which is modified below:

10. First tergite at most as long as wide at hind, usually slightly shorter; second tergite slightly shorter than, or sometimes as long as third tergite; head in dorsal view somewhat less rounded behind eyes; hind coxa always reddish yellow.

C. kariyai (Watanabe,1937)

- $\quad$ First tergite usually slightly longer than wide at hind

A. Second tergite one-fourth to one-fifth shorter than third tergite; third tergite usually more or less and fourth tergite less usually with yellow to reddish yellow pattern; tegulae yellow to vivid yellow

C. ruficrus (Haliday, 1834)

- Second tergite 1.2-1.3 $\times$ as long as third tergite; third and fourth tergite yellowish brown (Fig. 1E); tegulae yellow. C. elongata Zargar \& Gupta sp. nov. 
Cotesia khuzestanensis Zargar \& Gupta sp. nov. urn:lsid:zoobank.org:act:06139172-5335-4B37-8DE1-870B9D598879

Fig. 2

\section{Diagnosis}

Penultimate segment of antenna $1.5 \times$ as long as wide; mesoscutum densely evenly punctate to rugose (Fig. 2C); notauli densly punctate; scutellum punctate, interspaces as wide as diameter of punctation (Fig. 2C); metacoxa punctate and rugulose; pterostigma $3 \times$ as long as wide, brown (Fig. 2D); 1-R1 $1.3 \times$ as long as pterostigma, $3.5 \times$ as long as distance from end of $1-\mathrm{R} 1$ to tip of radial cell; first tergite parallel sided, $1.3 \times$ longer than apical width, rugose (Fig. $2 \mathrm{E}$ ).

\section{Etymology}

The name khuzestanensis refers to the name of Khuzestan Province in southwestern Iran, which is also the distribution range of this species.

\section{Material examined}

\section{Holotype}

IRAN • O; Khuzestan Province, Lali, Taraz; 32²0'49.70" N, 4905'11.31" E; 390 m a.s.1.; 4-18 May 2016; M. Zargar leg.; Malaise trap; citrus orchards; ICAR-NBAIR/NIM/MICROG/COT/45161H.

\section{Paratypes}

IRAN • 3 q $O$; same collection data as for holotype; TMUC-HBMC0045-0047.

\section{Description}

Female

MEAsurements. Body length $2.5 \mathrm{~mm}$, fore wing length $2.5 \mathrm{~mm}$.

HEAD (Fig. 2A-B). Smooth except with shallow punctations on face; width of head in dorsal view $1.8 \times$ as long as height; width of face as long as height; POL:MOD:OOL $5: 2: 4.4$; gena $0.6 \times$ as long as width of eye; malar space $1.3 \times$ as long as width of mandibular base; antenna as long as body; flagellomeres finely setose. FL1 $1_{1 / \mathrm{w}}: 3, \mathrm{FL}_{1 / \mathrm{w}}: 1.9, \mathrm{FL}_{1 / \mathrm{w}}: 1.5, \mathrm{FL}_{1 / \mathrm{w}}: 1.5, \mathrm{FL}_{1 / \mathrm{w}}: 1.4-1.5$.

Mesosoma (Fig. 2C). Mesoscutum densely evenly punctate to rugose, dull; notauli indicated by dense punctations, posteriorly merging to rugose sculpture; scutellum punctate and shiny, interspace as wide as diameter of punctate; scutellar sulcus crenulate; postscutellum crenulate and dull; prepectal carina absent; propodeum coarsely rugose to scabrous.

Wings (Fig. 2D). Areolet absent, vein $\mathrm{r}$ arising little after middle of pterostigma; $1-\mathrm{R} 11.3 \times$ as long as pterostigma, $3.5 \times$ as long as distance from end of $1-\mathrm{R} 1$ to tip of radial cell, $4.0 \times$ as long as $\mathrm{r}$; pterostigma $3.0 \times$ as long as wide; $r$ as long as $2-\mathrm{SR}$; $1-\mathrm{cu} 1$ as long as $2-\mathrm{cu} 1$; width of discoidal cell $1.1 \times$ as long as height.

LEGS (Fig. 2 F). Metacoxa $1.3 \times$ as long as first tergite; metafemur length $4.0 \times$ as long as median width; inner spur of metatibia slightly longer than outer, as long as half of basitarsus.

Metasoma (Fig. 2E). TI parallel sided, $1.3 \times$ as long as apical width, rugose. TII apical width $2.5 \times$ as long as median length, rugose. TIII median length $1.5 \times$ as long as TII; tergites posterior to TII smooth and shiny; ovipositor sheath $0.7 \times$ as long as metabasitarsus. Hypopygium $0.5 \times$ as long as metatibia, truncated apically. 

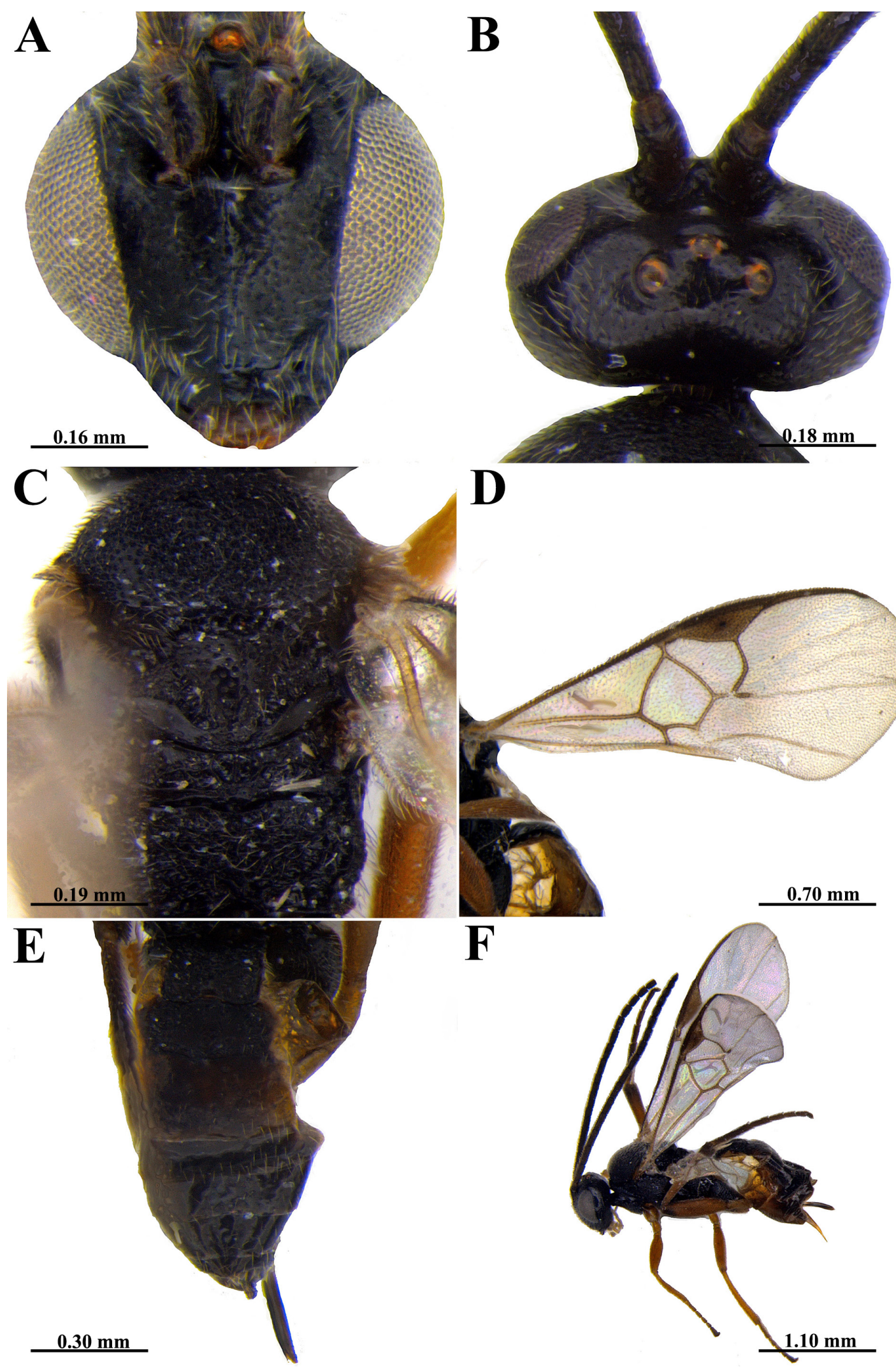

Fig. 2. Cotesia khuzestanensis Zargar \& Gupta sp. nov., ICAR-NBAIR/NIM/MICROG/COT/451611. A. Head, frontal view. B. Head, dorsal view. C. Mesosoma, dorsal view. D. Fore wing. E. Metasoma, dorsal view. F. Habitus, lateral view. 
CoLour. Antenna dark brown; head and mesosoma black; metasoma black except brown third tergite and basal sternites; labial palp and maxillary palp yellow; tegula yellowish brown; profemur and mesofemur reddish brown; metafemur reddish brown except black spot at apex; tibia reddish brown; protarsus and mesotarsus reddish brown; metatarsus dark brown; pterostigma brown.

\section{Male}

Unknown.

\section{Host}

Unknown.

\section{Biology}

Unknown.

\section{Remarks}

In the key provided by Nixon (1974) this new species runs close to Cotesia setebis (Nixon, 1974); it can, however, be separated by the following characters: (1) first tergite parallel-sided, $1.3 \times$ as long as apical width (Fig. 2E) vs as long as apical width in C. setebis, (2) inner spur of metatibia slightly longer than outer reaching middle of basitarsus (Fig. 2F) vs distinctly longer than outer one and longer than half of basitarsus, (3) metafemur reddish brown (Fig. 2F) vs black in C. setebis. In recent years, two species were described that are close to C. setebis: Cotesia adippevora Shaw, 2009 and C. acerbiae Shaw \& Vikberg, 2015. For both these species the length of the first tergite is as long as its apical width.

The new species can be included in the identification key of Cotesia compiled by Nixon (1974), which is modified below:

3. Inner spur of metatibia conspicuously to marginally longer than outer, clearly reaching or crossing

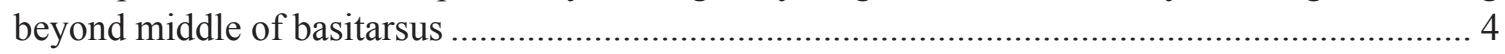

- Inner spur of metatibia not longer than outer and shorter than half of basitarsus ......................... 5

4. Preapical segment about twice as long as wide; mesoscutum on the whole coarsely rugosepunctate, without a contrasting dull area posteriorly.....

C. callimone (Nixon, 1974)

- Preapical segment about $1.5 \times$ as long as wide; mesoscutum on the whole dull, with punctation of variable intensity; notaulic bands uniting posteriorly to form a large zone of dull area

A. First tergite parallel-sided, $1.3 \times$ as long as its apical width (Fig. 2E); inner spur of metatibia slightly longer than outer, as long as half of basitarsus (Fig. 2F); metafemur reddish brown except black spot at apex (Fig. 2F)

C. khuzestanensis Zargar \& Gupta sp. nov.

- $\quad$ First tergite as long as apical width; inner spur distinctly longer than outer and slightly longer than half of hind basitarsus; metafemur black.

C. setebis (Nixon, 1974)

The new species runs in the key of Papp (1986) reaching to couplet number 34 which was later modified (Papp 1987). Further modifications to fit this species in Papp (1987) are suggested as below:

34. Inner spur of metatibia shorter than half basitarsus; first tergite distinctly broadening posteriorly, about as long as wide at hind; head in dorsal view less transverse, 1.8 times as broad as long......

C. analis (Nees, 1834)

- Inner spur of metatibia as long as or longer than half basitarsus.

A. First tergite as long as wide at hind .......C. setebis (Nixon, 1974) and C. callimone (Nixon, 1974)

- First tergite $1.3-1.5 \times$ as long as wide at hind ..... 
B. Inner spur of metatibia slightly longer than outer, as long as half of basitarsus (Fig. 2F); penultimate segment of antenna $1.4 \times$ as long as wide; $r$ oblique to fore margin of pterostigma (Fig. 2D) C. khuzestanennsis Zargar \& Gupta sp. nov.

- Inner spur of metatibia distinctly longer than half of basitarsus; penultimate segment of antenna $2.0 \times$ as long as wide. C. setebis (Nixon, 1974) and C. callimone (Nixon, 1974)

Cotesia zagrosensis Zargar \& Gupta sp. nov.

urn:1sid:zoobank.org:act:2E7929F1-339D-4354-A27A-80F87E99EB7B

Fig. 3

\section{Diagnosis}

Penultimate segment of antenna $1.5 \times$ as long as wide; mesoscutum subshiny, more or less with traces of punctatations (Fig. 3C); scutellum smooth and shiny; pterostigma $2.6 \times$ as long as wide, light brown (Fig. 3D); first tegite sub-parallel sided, strongly rounded apically, $1.3 \times$ as long as apical width, smooth, light brown (Fig. 3E); apical width of second tergite $4 \times$ as long as median length, smooth, light brown (Fig. 3E); third tergite $1.6 \times$ as long as second tergite (Fig. 3E).

\section{Etymology}

The name refers to the Zagros mountain range, which is also the distribution range of the species.

\section{Material examined}

\section{Holotype}

IRAN • + ; Khuzestan, Lali, Taraz; 32²0'49.70" N, 4905'11.31" E; 390 m a.s.1.; 6-20 Jul. 2017; Malaise trap; citrus orchards; M. Zargar leg.; ICAR-NBAIR/NIM/MICROG/COT/6717H.

\section{Paratype}

IRAN • 1 क ; same collection data as for holotype; TMUC-HBMC0048.

\section{Description}

\section{Female}

Measurement. Body length $2.5 \mathrm{~mm}$, fore wing length $2.5 \mathrm{~mm}$.

HEAD (Fig. 3A-B). Smooth except shallowly punctate on face; width of head in dorsal view $1.8 \times$ as long as height; width of face $1.3 \times$ as long as height; POL:MOD:OOL $4.1: 2: 3.5$; gena $0.4 \times$ as long as width of eyes; malar space $1.3 \times$ as long as width of mandibular base; antenna shorter than body; flagellomeres finely setose. FL $1_{1 / \mathrm{w}}: 2.8, \mathrm{FL}_{1 / 1 / \mathrm{w}}: 1.6, \mathrm{FL}_{1 / \mathrm{w}}: 1.5, \mathrm{FL}_{1 / 1}: 1.5, \mathrm{FL} 15_{1 / \mathrm{w}}: 1.5$.

Mesosoma (Fig. 3C). Mesoscutum subshiny more or less with traces of punctatations; notauli indistinct; scutellum smooth and shiny; scutellar sulcus crenulate; postscutellum crenulate and shiny; prepectal carina absent; propodeum rugose with median carina.

WiNGS (Fig. 3D). Areolet absent, vein $\mathrm{r}$ arising from middle of pterostigma; $1-\mathrm{R} 11.3 \times$ as long as pterostigma, $3.5 \times$ as long as distance from end of $1-\mathrm{R} 1$ to tip of radial cell, $3.7 \times$ as long as $\mathrm{r}$; pterostigma $2.6 \times$ as long as wide; $\mathrm{r} 1.4 \times$ as long as $2-\mathrm{SR} ; 1-\mathrm{cu} 10.8 \times$ as long as $2-\mathrm{cu} 1$; width of discoidal cell $1.2 \times$ as long as height.

LeGs (Fig. 3F). Metacoxa $1.5 \times$ as long as first tergite; metafemur length $3.1 \times$ as long as median width; inner and outer spur of metatibia equal, $0.4 \times$ as long as basitarsus. 


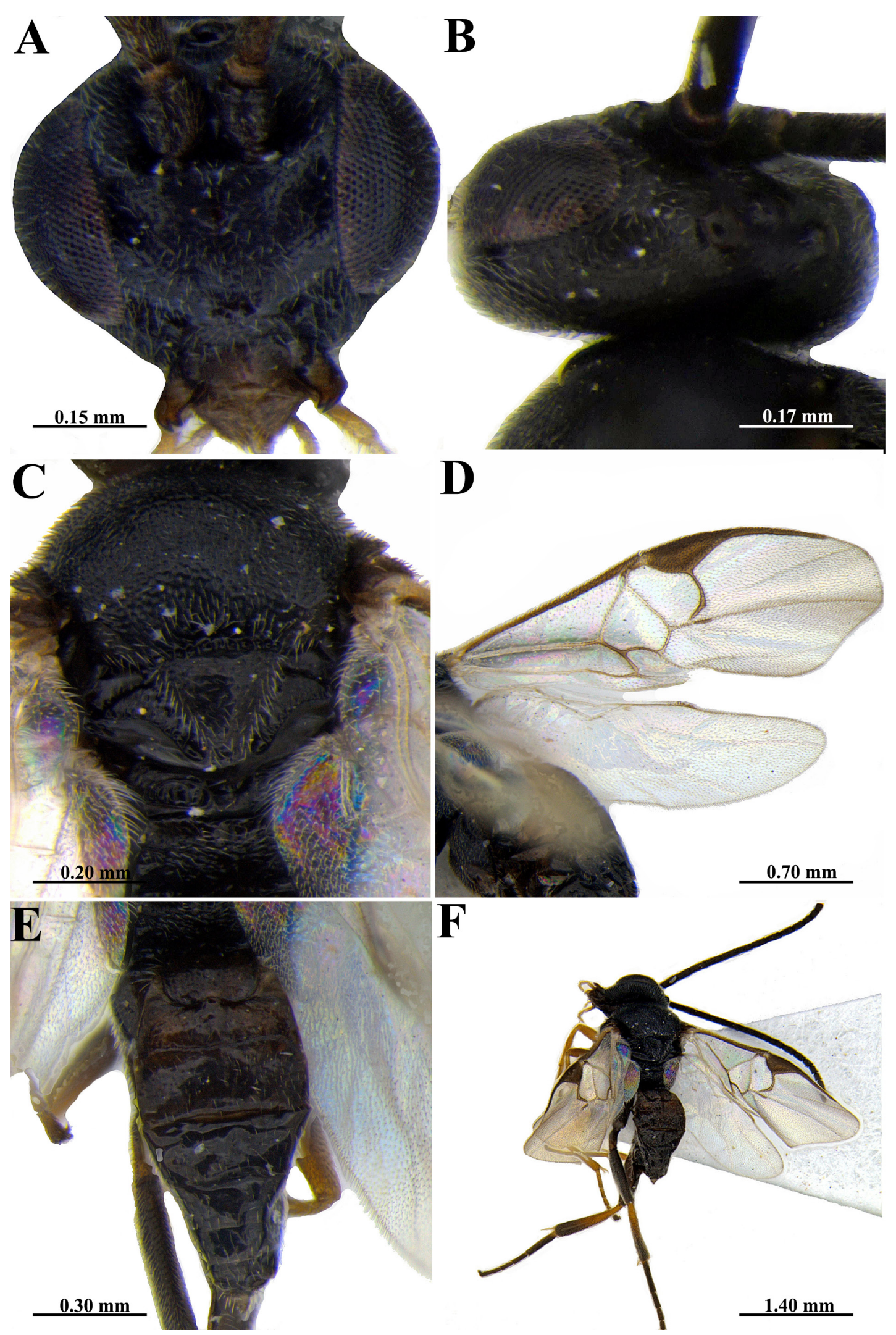

Fig. 3. Cotesia zagrosensis Zargar \& Gupta sp. nov., ICAR-NBAIR/NIM/MICROG/COT/6717. A. Head, frontal view. B. Head, dorsal view. C. Mesosoma, dorsal view. D. Fore wing. E. Metasoma, dorsal view. F. Habitus, dorsal view. 
Metasoma (Fig. 3E). TI parallel sided, $1.3 \times$ as long as apical width, shallowly rugolose at apex near hind margin, remaining smooth, shiny. TII apical width $4 \times$ as long as median length, smooth. TIII median length $1.6 \times$ as long as TII; tergites posterior to TI smooth and shiny; ovipositor sheath $0.7 \times$ as long as metabasitarsus; hypopygium $0.7 \times$ as long as metatibia, truncated apically.

Colour. Antenna dark brown; head, mesosoma black; metasoma, first and second tergite light brown, third tergite dark brown, remaining black; labial palp and maxillary palp yellow; tegula dark brown; profemur reddish brown, mesofemur dark brown in anterior half, reddish brown in posterior half; metafemur dark brown; protibia, mesotibia yellowish brown, metatibia reddish brown except black infuscations at apex; protarsus and mesotarsus yellowish brown; metatarsus dark brown. Pterostigma light brown.

\section{Male}

Unknown.

\section{Host}

Unknown.

\section{Biology}

Unknown.

\section{Distribution}

Khuzestan Province (Iran).

\section{Comments}

In the key of Nixon (1974) the new species runs smoothly to Cotesia onaspis (Nixon, 1974) from which it can be easily separated by: (1) second tergite conspicuously transverse and $4 \times$ as long as median length (Fig. 3E) vs $2.5 \times$ in C. onaspis, (2) phragma of scutellum not visible (Fig. 3C) vs narrowly visible in C. onaspis, (3) metafemur dark brown (Fig. 3F) vs reddish yellow in C. onaspis.

The new species can be included in the identification key of Cotesia compiled by Nixon (1974), which is modified below:

55. Gaster blackish; first tergite (as also basal field) dull, densely rather finely rugose all over; metacarp hardly three times as long as its distance from apex of radial cell. Basella meeting submediella almost at right-angle.....

C. notha (Marshall, 1885)

- Gaster frequently pale brownish with tergites 3-5 showing broad dark apical bands; first tergite shiny, sculpture of apical part consisting of coarse rugose-punctation that tends to fade out along middle line; metacarp nearly four times as long as its distance from apex of radial cell. Basella curved; fringe of vannal lobe hardly noticeable

A. Second tergite conspicuously transverse, 4.0 times as long as median length (Fig. 3E); phragma of scutellum not visible (Fig. 3C); metafemur dark brown (Fig. 3F)

C. zagrosensis Zargar \& Gupta sp. nov.

- Second tergite less transverse, 2.5 times as long as median length; phragma of scutellum narrowly visible; metafemur reddish yellow

C. onaspis (Nixon, 1974)

In Papp's key, this species runs to Cotesia notha (Marshall, 1885). The key can be modified as follows:

175. Second tergite conspicuously less transverse, 1.9-2.2 times, usually twice as wide as long medially; first tergite sometimes less broadening posteriorly; pterostigma rather wide, 2-2.3 times as long as wide ..C. juniperatae (Bouché,1834) [valid name: C. sessilis (Geoffroy,1785)]

- $\quad$ Second tergite transverse, at least 2.5 times as wide as long medially. ..A 
A. Metacarp relatively short, about thrice as long as distance between its distal end and tip of radial cell; first tergite less rounded behind; nervellus (of hind wing) almost straight; metafemur black or blackish, sometimes light brown to brownish yellow, rarely with rusty suffusion C. notha (Marshall, 1885)

- Metacarp long, 3.7 as long as distance between its distal end and tip of radial cell (Fig. 3D); first tergite strongly rounded behind (Fig. 3E); nervellus curved; metafemur dark brown (Fig. 3F) C. zagrosensis Zargar \& Gupta sp. nov.

\section{New records of Cotesia from Iran}

\section{Cotesia cynthiae (Nixon, 1974)}

Fig. 4

Apanteles cynthiae Nixon, 1974: 499.

\section{Diagnosis}

Antenna $1.1 \times$ as long as body, penultimate segment of antenna $2.0 \times$ as long as wide; width of face $1.2 \times$ as long as height (Fig. 4A); POL:MOD:OOL 25:13:25; width of head in dorsal view $1.9 \times$ as long as height (Fig. 4B); mesonotum coarsely reticulate-punctate to rugose (Fig. 4C); scutellum rugosepunctate; $1-\mathrm{R} 11.5 \times$ as long as pterostigma, $3.0 \times$ as long as distance from end of $1-\mathrm{R} 1$ to tip of radial cell; pterostigma $3.2 \times$ as long as wide (Fig. 4D); metacoxa coarsely rugose; metasoma posteriorly compressed laterally (Fig. 4F); first tergite broadening posteriorly, medial length as long as apical width, rugose; second tergite as long as third tergite, rugose (Fig. 4E); ovipositor sheath $0.3 \times$ as long as metabasitarsus; hypopygium $0.5 \times$ as long as metatibia (Fig. 4F); legs reddish brown; body black except third tergite.

\section{Material examined}

IRAN - Khuzestan Province • 3 q ; ; Lali, Taraz; 32²0'49.70" N, 4905'11.31" E; 390 m a.s.1.; 4-18 Mar. 2016, 3-17 Apr. 2016; Malaise trap; citrus orchards; M. Zargar leg.; TMUC-HBMC0049-0051 • 1 ; s same collection data as for preceding; 3-17 Apr. 2016; ICAR-NBAIR/NIM/MICROG/COT/3416.

\section{Distribution in Iran}

Khuzestan (present study). New record for Iran.

\section{General distribution}

Austria, Bulgaria, France, Hungary, Switzerland, Turkey (Yu et al. 2016).

\section{Host record}

Lepidoptera: Nymphalidae Rafinesque, 1815 (Yu et al. 2016).

\section{Cotesia glabrata (Telenga, 1955)}

Fig. 5

Apanteles glabratus Telenga, 1955: 132.

\section{Diagnosis}

Antenna shorter than body 0.8 , penultimate segment $1.4 \times$ as long as wide; width of face $1.4 \times$ as long as height (Fig. 5A); POL:MOD:OOL 15:6:11; width of head in dorsal view $2.1 \times$ as long as height 


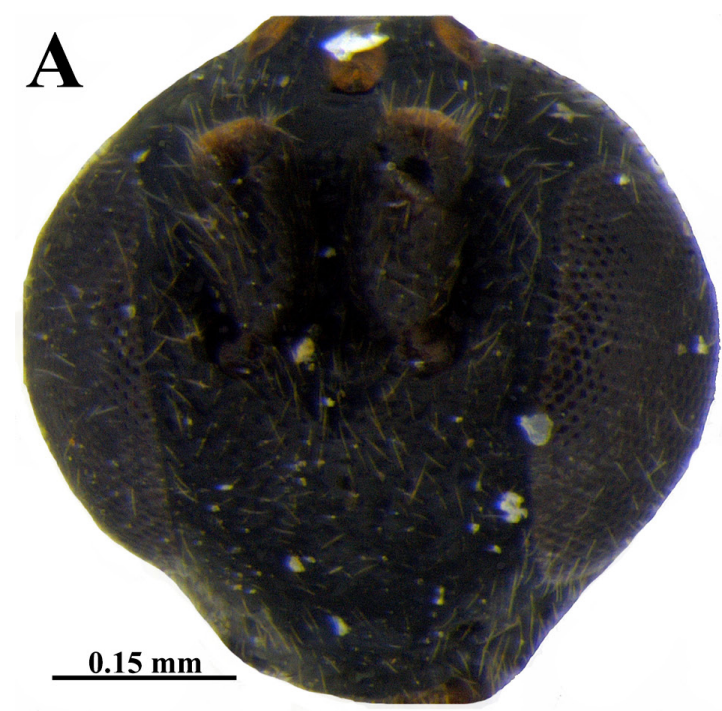

\section{B}

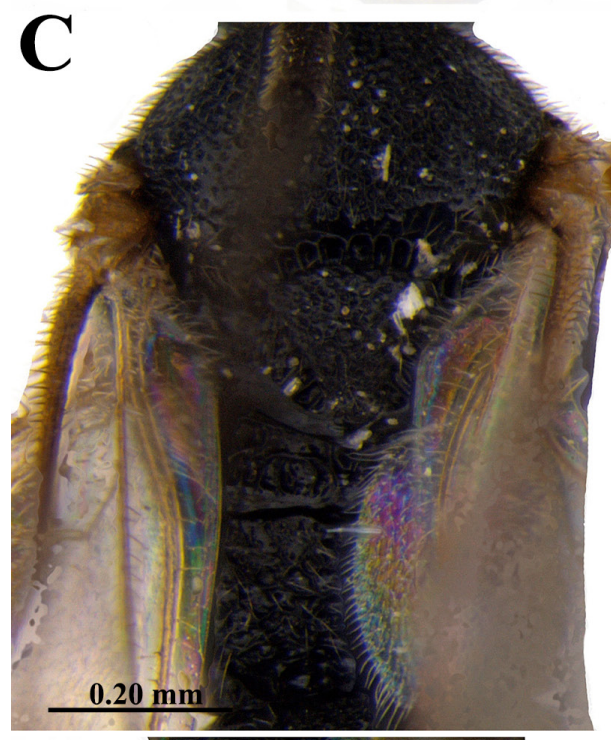

E
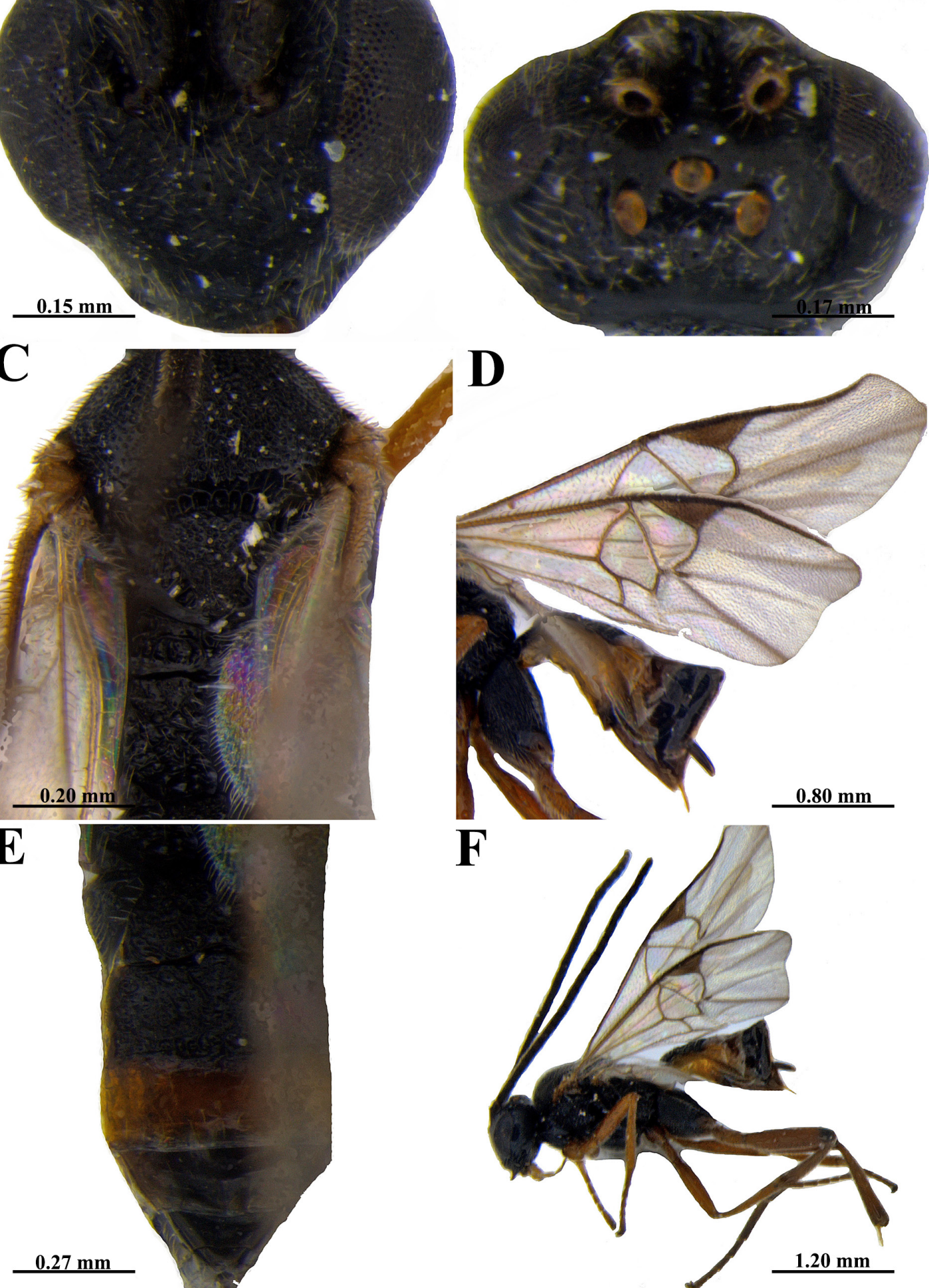

$\mathbf{F}$

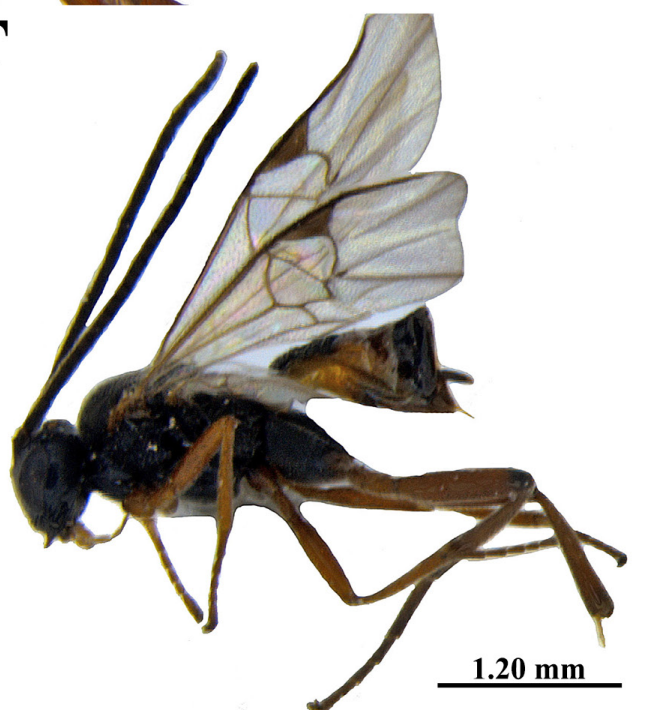

Fig. 4. Cotesia cynthiae (Nixon, 1974). A. Head, frontal view., ICAR-NBAIR/NIM/MICROG/ COT/3416. B. Head, dorsal view. C. Mesosoma, dorsal view. D. Fore wing. E. Metasoma, dorsal view. F. Habitus, lateral view. 

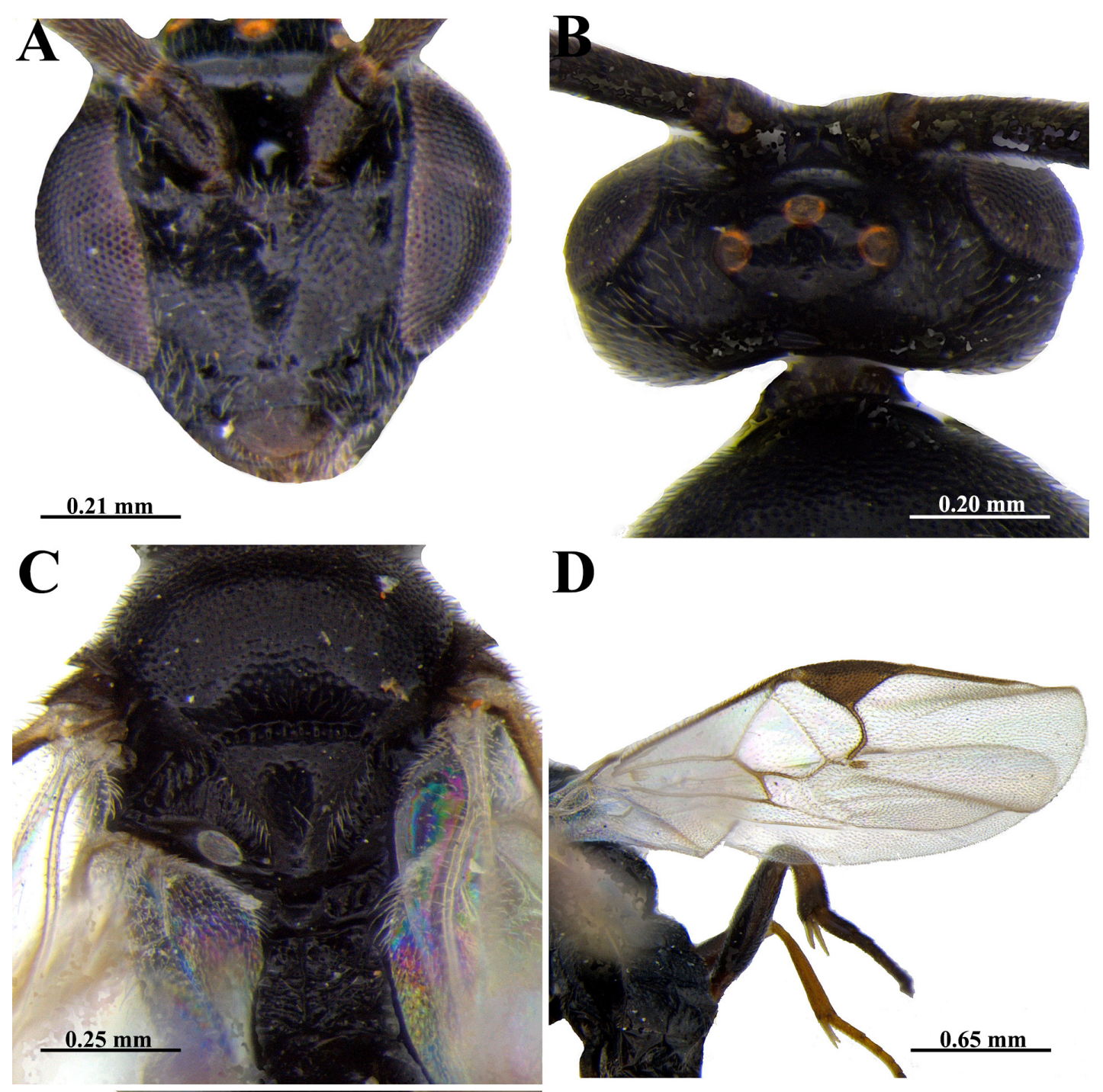

D
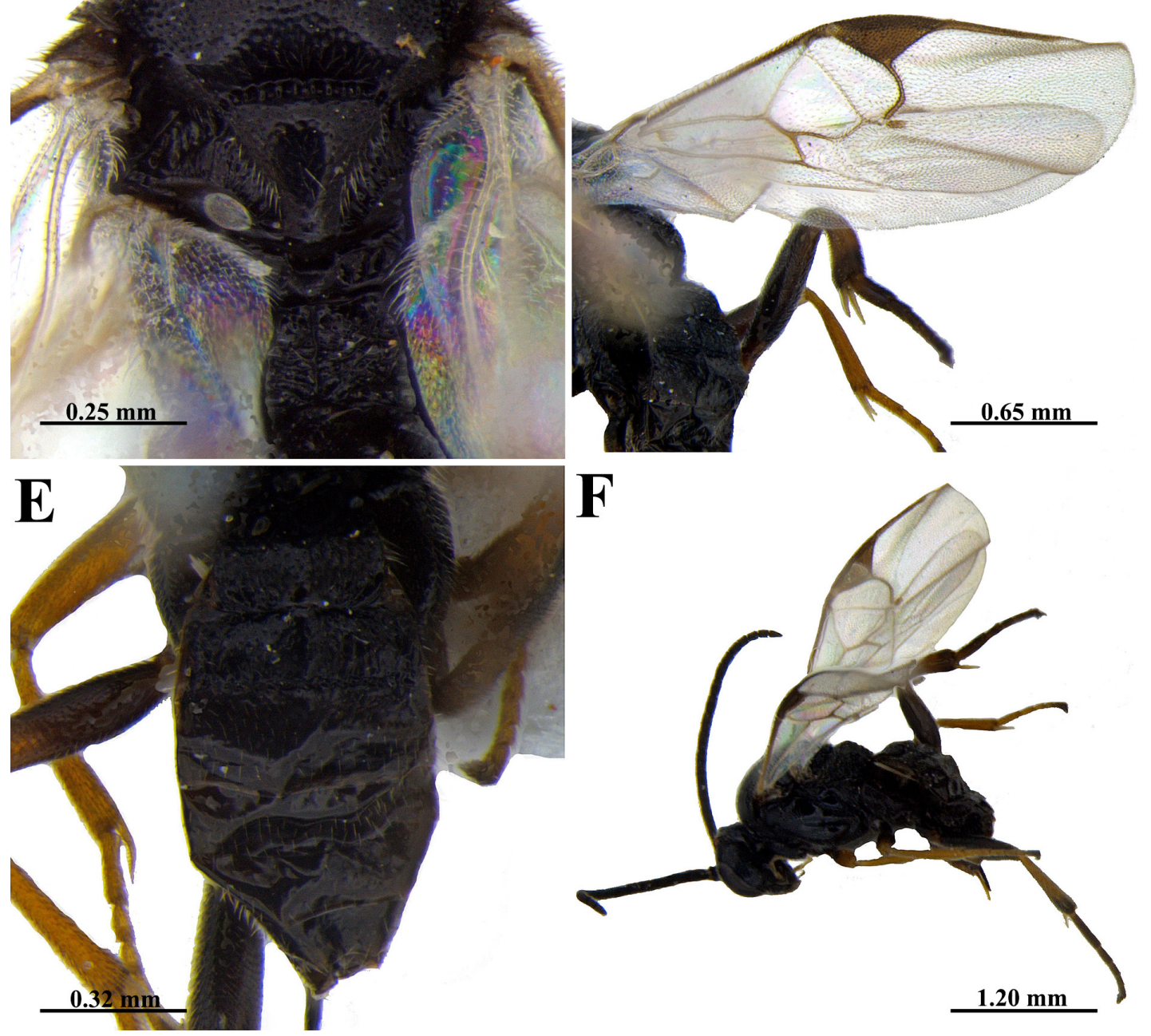

Fig. 5. Cotesia glabrata (Telenga, 1955), ICAR-NBAIR/NIM/MICROG/COT/20416. A. Head, frontal view. B. Head, dorsal view. C. Mesosoma, dorsal view. D. Fore wing. E. Metasoma, dorsal view. F. Habitus, lateral view. 
(Fig. 5B); mesonotum shallowly punctuate, shiny; scutellum smooth, shiny (Fig. 5C); 1-R1 1.3× as long as pterostigma, $3.4 \times$ as long as distance from end of $1-\mathrm{R} 1$ to tip of radial cell ; pterostigma $2.4 \times$ as long as wide (Fig. 5D); metacoxa smooth; first tergite distinctly broader posteriorly, medial length $0.8 \times$ as long as apical width, rugose; second tergite in middle smooth and laterally rugose, $3 \times$ wider than long medially (Fig. 5E); ovipositor sheath $0.5 \times$ as long as metabasitarsus; hypopygium $0.6 \times$ as long as metatibia (Fig. 5F); body black.

\section{Material examined}

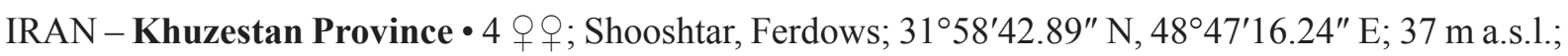
20 Apr.-4 May 2016, 5-19 Jun. 2017, 22 Jun.-6 Jul. 2017; Malaise trap; citrus orchards; M. Zargar leg.; TMUC-HBMC0052-0055 - 1 क; same collection data as for preceding; 20 Apr-4 May 2016; ICARNBAIR/NIM/MICROG/COT/20416.

\section{Distribution in Iran}

Khuzestan (present study). New record for Iran.

\section{General distribution}

Bulgaria, Georgia, Germany, Hungary, Israel, Kazakhstan, Russia, Turkey, Ukraine (Yu et al. 2016).

\section{Host records}

Lepidoptera: Geometridae Leach, 1815, Hesperiidae Latreille, 1809, Pieridae Swainson, 1820 (Yu et al. 2016).

\section{New records of Cotesia from Khuzestan Province of Iran}

\section{Cotesia jucunda (Marshall, 1885)}

Apanteles jucundus Marshall, 1885: 182.

Microgaster nigrinervis Thomson, 1895: 2260.

\section{Material examined}

IRAN - Khuzestan Province • 1 9; Andika, Doorab; $32^{\circ} 12^{\prime 2} 23.00^{\prime \prime}$ N, 49²6'37.00" E; 760 m a.s.1.; 6-20 Mar. 2017; TMUC-HBMC0056 • 1 क ; Chezi; 3208'02.78" N, 49³8'30.56" E; 650 m a.s.1.; 4-20 May 2016; ICAR-NBAIR/NIM/MICROG/COT/4516 • 2 우; Lali, Taraz; 32²0'49.70" N, 4905'11.31" E; 390 m a.s.1.; 5-19 May 2017, 5-19 Jun. 2017; TMUC-HBMC0057-0058 • 1 क; Dobagh; 31 ${ }^{\circ} 31^{\prime} 16.14^{\prime \prime} \mathrm{N}$, 4952'53.00" E; $688 \mathrm{~m}$ a.s.1.; 4-18 May 2016; Malaise trap; citrus orchards; M. Zargar leg.; TMUCHBMC0059.

\section{Distribution in Iran}

Guilan (Ghahari et al. 2012a), Markazi (Ghahari et al. 2011d), Khuzestan (present study).

\section{General distribution}

Armenia, Austria, Bulgaria, Croatia, Czechoslovakia, Denmark, Estonia, Finland, France, Germany, Greece, Hungary, Iran, Ireland, Moldova, Mongolia, Poland, Romania, Russia, Serbia, Spain, Sweden, Switzerland, Turkey, United Kingdom, Yugoslavia (Yu et al. 2016).

\section{Host records}

Lepidoptera: Geometridae (Shenefelt 1972; Capek 1972; Yu et al. 2016), Nymphalidae, Pieridae (Yu et al. 2016). 
Cotesia praepotens (Haliday, 1834)

Microgaster praepotens Haliday, 1834: 252.

Microgaster placida Haliday, 1834: 251.

Apanteles memnon Nixon, 1974: 465, fig. 39.

Apanteles acutivalvis Balevski, 1980: 97.

Apanteles beshtaui Tobias, 1986: 716, fig. 232:18.

\section{Material examined}

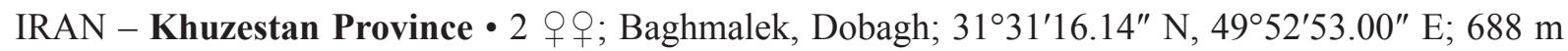
a.s.1.; 4-18 May 2016; ICAR-NBAIR/NIM/MICROG/COT/4516 • 1 q; Ghale tol; 31 ${ }^{\circ} 37^{\prime} 49.70^{\prime \prime} \mathrm{N}$, 49 52'53.35" E; 880 m a.s.1.; 22 Jun.-6 Jul. 2017; ICAR-NBAIR/NIM/MICROG/COT/22617 • 1 क; Andika, Doorab; 32¹2'23.00" N, 49²6'37.00" E; 760 m a.s.1.; 5-19 May 2017; TMUC-HBMC0060 •

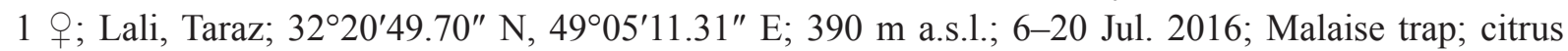
orchards; M. Zargar leg.; TMUC-HBMC0061.

\section{Distribution in Iran}

Qazvin (Ghahari et al. 2011c), Khuzestan (present study).

\section{General distribution}

Afghanistan, Armenia, Azerbaijan, Bulgaria, Croatia, Czech Republic, Finland, Germany, Greece, Hungary, Iran, Ireland, Italy, Kazakhstan, Lithuania, Macedonia, Moldoava, Mongolia, Poland, Romania, Russia, Slovakia, Spain, Sweden, Switzerland, Tajikistan, Turkey, Turkmenistan, United Kingdom, Uzbekistan, Yugoslavia (Yu etal. 2016).

\section{Host records}

Lepidoptera: Elachistidae Bruand, 1851 (Yu et al. 2016), Geometridae (Shenefelt 1972; Nixon 1974; Yu et al. 2016), Gracillariidae Stainton, 1854, Lymantriidae Hampson, 1893, Notodontidae Hampson, 1893, Yponomeutidae Stephens, 1829 (Yu et al. 2016).

\section{Cotesia risilis (Nixon, 1974)}

Apanteles risilis Nixon, 1974: 471, fig. 27.

\section{Material examined}

IRAN - Khuzestan Province • 1 q; Baghmalek, lalab; 31³2'53.70" N, 4957'37.00" E; 843 m a.s.l.; 6-20 Mar. 2017; Malaise trap; citrus orchards; M. Zargar leg.; TMUC-HBMC0062.

\section{Distribution in Iran}

Ilam (Ghahari et al. 2011b), West Azarbaijan (Samin 2015), Khuzestan (present study).

\section{General distribution}

Czechoslavia, Greece, Hungary, Iran, Italy, Mongolia, Montenegro, the Netherlands, Romania, Slovakia, Turkey, United Kingdom, Yugoslavia (Yu et al. 2016).

\section{Host records}

Lepidoptera: Pieridae (Nixon 1974; Yu et al. 2016). 
Cotesia vestalis (Haliday, 1834)

Microgaster vestalis Haliday, 1834: 253.

Apanteles plutellae Kurdjumov, 1912: 226.

\section{Material examined}

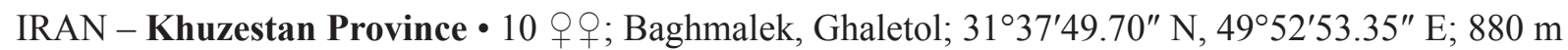
a.s.1.; 4-18 May 2016, 4-18 Jun. 2016, 5-19 Jun. 2017, 22 Jun.-6 Jul. 2017; TMUC-HBMC0063-0072 • 1 ; ; same collection data as for preceding; 3-17 Apr. 2016; ICAR-NBAIR/NIM/MICROG/COT/3416 • 1 \%; same collection data as for preceding; 4-18 May 2016; ICAR- NBAIR/NIM/MICROG/COT/4516 • 12 우; Shang; 3131'46.00" N, 4953'14.61" E; 716 m a.s.1.; 20 Mar.-4 Apr. 2016, 4-18 May 2016, 4-18 Apr. 2017, 5-19 May 2017, 4-18 Jul. 2017; TMUC-HBMC0073-0084 • 11 qo+; Dobagh; 31 ${ }^{\circ} 31^{\prime} 16.14^{\prime \prime} \mathrm{N}, 49^{\circ} 52^{\prime} 53.00^{\prime \prime}$ E; 688 m a.s.1.; 3-17 Apr. 2016, 3-17 May 2016, 4-18 Jun. 2017; Malaise trap; citrus orchards; TMUC-HBMC0085-0095 - 11 q ; Behbahan, Dodangeh; 30 42'08.38" N, 50¹0'41.81" E; 300 m a.s.1.; 4-18 Apr. 2016, 3-17 Jun. 2016, 5-19 Mar. 2017, 5-19 May 2017, 22 Jun.-6 Jul 2017; Malaise trap; palm orchards; TMUC-HBMC0096-0106 • 9 우; Dezful, Qaleh-ye Rob-e Bandbal; 32¹7'27.94" N, 48²5'46.98" E; 97 m a.s.1.; 3-17 May 2016, 22 May-6 Jun. 2017, 4-18 Jun. 2017; TMUC-HBMC0107-0115 • 16 q ; ; Shamsabad; 32²9'64.65" N, 4842'57.45" E; 94 m a.s.1.; 3-17 Apr. 2016, 3-17 May 2016, 22 May-6 Jun. 2017; TMUC-HBMC0116-0131 •

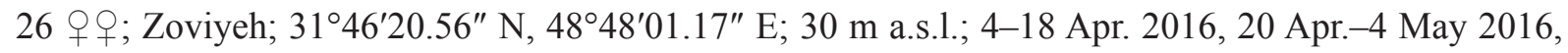
22 May-6 Jun. 2016, 5-19 Jun. 2017, 22 Jun.-6 Jul. 2017; Malaise trap; citrus orchards; TMUCHBMC0132-0157 • 12 우; Khoramshahr, Sheneh; 30²5'32.24" N, 48¹1'20.83" E; 2 m a.s.1.; 20 Apr.-4 May 2016, 4-18 Jun. 2016, 4-18 Apr. 2017, 5-19 Jun. 2017, 22 Jun.-6 Jul. 2017; Malaise trap;

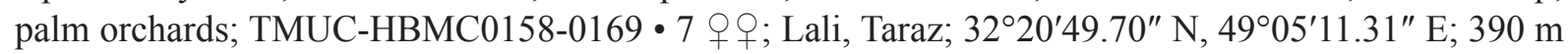
a.s.1.; 3-17 Apr. 2016, 4-18 May 2016, 5-19 Mar. 2017, 5-19 May 2017, 22 Jun.-6 Jul. 2017; TMUCHBMC0170-0176 • 7 우; Andika, Chezi; 3208'02.78" N, 49³8'30.56" E; 650 m a.s.1.; 20 Apr.-4 May 2016, 4-18 Jun. 2016, 22 May-6 Jun. 2017, 22 Jun.-6 Jul. 2017; TMUC-HBMC0177-0183 • 9 우; Doorab; 32¹2'23.00" N, 49²6'37.00" E; 760 m a.s.1.; 20 Apr.-4 May 2016, 4-18 Jun. 2016, 4-18 Apr. 2017, 22 Jun.-6 Jul. 2017; Malaise trap; citrus orchards; TMUC-HBMC0184-0192 - 7 우; Ramhormoz, Gharabad; 3059'37.73" N, 4946'50.63" E; 126 m a.s.1.; 5-19 Jun. 2017, 22 Jun.-6 Jul. 2017; Malaise trap; olive orchards; M. Zargar leg.; TMUC-HBMC0193-0199.

\section{Distribution in Iran}

Alborz (Golizadeh et al. 2007), East Azarbaijan (Rastegar et al. 2012), Isfahan (Ghahari et al. 2011a), Tehran (Hassanshahi et al. 2012), Khuzestan (present study).

\section{General distribution}

Afghanistan, Argentina, Armenia, Azerbaijan, Bangladesh, Belgium, Benin, Brazil, Bulgaria, Cape Verde Islands, China, Czech Republic, Finland, France, Germany, Greece, Hungary, India, Iran, Ireland, Israel, Italy, Japan, Kazakhstan, Kenya, Korea, Kyrgyzstan, Latvia, Libya, Macedonia, Malaysia, Moldova, Mongolia, Morocco, the Netherlands, New Zealand, Pakistan, Papua New Guinea, Philippines, Poland, Portugal, Romania, Russia, Serbia, Singapore, South Africa, Spain, Sri Lanka, Sweden, Switzerland, Tajikistan, Thailand, Turkey, Turkmenistan, USA, Ukraine, UK, Uzbekistan, Vietnam, former Yugoslavia (Yu et al. 2016); Australia (Shenefelt 1972); Réunion (Rousse \& Gupta 2013).

\section{Host records}

Lepidoptera: Arctiidae Leach, 1815 (Yu et al. 2016), Lasiocampidae Harris, 1841 (Nixon 1974; Yu et al. 2016), Lymantriidae Hampson, 1893 (Shenefelt 1972; Yu et al. 2016), Nymphalidae, Pieridae, 
Table 1 (continued on next page). Updated list of Cotesia Cameron, 1891 species occurring in Iran.

\begin{tabular}{|c|c|c|}
\hline Species of Cotesia & Distribution in Iran (Province) & References \\
\hline C. abjecta (Marshall, 1885) & Lorestan & Ghahari et al. $2012 \mathrm{a}$ \\
\hline C. ancilla (Nixon, 1974) & Isfahan, Kermanshah & $\begin{array}{l}\text { Ghahari et al. 2011a; Ghahari \& Fischer } \\
2012\end{array}$ \\
\hline C. callimone (Nixon, 1974) & Lorestan, Mazandaran, Semnan & $\begin{array}{l}\text { Ghahari et al. 2012a; Sakenin et al. 2012; } \\
\text { Naderian et al. } 2012\end{array}$ \\
\hline C. chilonis (Munakata, 1910) & Not defined & Rassipour 1983; Modarres Awal 1997 \\
\hline C. cuprea (Lyle, 1925) & Lorestan & Ghahari et al. $2012 \mathrm{a}$ \\
\hline C. cynthiae (Nixon, 1974) & Khuzestan & Present study \\
\hline C. elongata Zargar \& Gupta sp. nov. & Khuzestan & Present study \\
\hline C. euryale (Nixon, 1974) & Ilam & Ghahari et al. 2011b \\
\hline C. flavipes Cameron, 1891 & Mazandaran & Ghahari et al. 2009 \\
\hline C. geryonis (Marshall, 1885) & Kermanshah & Ghahari \& Fischer 2012 \\
\hline C. glabrata (Telenga, 1955) & Khuzestan & Present study \\
\hline C. glomerata (Linnaeus, 1758) & Fars, Guilan, West Azarbaijan & $\begin{array}{l}\text { Lashkari Bod et al. 2011; Ghahari et al. } \\
\text { 2012b; Alizadeh \& Moghaddam 2004; } \\
\text { Khanjani 2006; Razmi et al. } 2011\end{array}$ \\
\hline C. hyphantriae (Riley, 1887) & Qazvin & Ghahari et al. 2011c \\
\hline C. jucunda (Marshall, 1885) & Guilan, Khuzestan, Markazi & $\begin{array}{l}\text { Ghahari et al. 2012a; present study; } \\
\text { Ghahari et al. 2011d }\end{array}$ \\
\hline C. kazak (Telenga, 1949) & Tehran & $\begin{array}{l}\text { Davatchi \& Chodjai 1969; Modarres } \\
\text { Awal } 1997\end{array}$ \\
\hline C. khuzestanensis Zargar \& Gupta sp. nov. & Khuzestan & Present study \\
\hline C. melanoscela (Ratzeburg, 1844) & East Azarbaijan & Ghahari et al. 2010 \\
\hline C. notha (Marshall, 1885) & Golestan, Lorestan & $\begin{array}{l}\text { Ghahari et al. } 2011 \mathrm{~b} \text {; } \\
\text { Ghahari et al. } 2012 \mathrm{a}\end{array}$ \\
\hline C. ofella (Nixon, 1974) & Mazandaran, West Azarbaijan & Ghahari et al. 2010; Karimpour et al. 2001 \\
\hline C. ordinaria (Ratzeburg, 1844) & Golestan, Lorestan & Sakenin et al. 2012; Ghahari et al. 2012a \\
\hline C. praepotens (Haliday, 1834) & Khuzestan, Qazvin & Present study; Ghahari et al. 2011c \\
\hline C. risilis (Nixon, 1974) & Ilam, Khuzestan, West Azarbaijan & $\begin{array}{l}\text { Ghahari et al. 2011b; Present study; } \\
\text { Samin } 2015\end{array}$ \\
\hline C. rubecula (Marshall, 1885) & Hamadan & Khanjani 2006 \\
\hline C. ruficrus (Haliday, 1834) & $\begin{array}{l}\text { Fars, Mazandaran Sistan and } \\
\text { Baluchestan }\end{array}$ & $\begin{array}{l}\text { Lashkari Bod et al. 2011; Abbasipour \& } \\
\text { Taghavi 2002; 2004; Khajeh et al. } 2014\end{array}$ \\
\hline C. salebrosa (Marshall, 1885) & Kermanshah & Ghahari \& Fischer 2012 \\
\hline C. saltator (Thunberg, 1922) & Sistan and Baluchestan & Khajeh et al. 2014 \\
\hline C. scabricula (Reinhard, 1880) & Guilan & Ghahari et al. $2012 \mathrm{~b}$ \\
\hline C. setebis (Nixon, 1974) & Golestan & Ghahari et al. 2011d \\
\hline C. sessilis (Geoffroy, 1785) & Ardabil, Mazandaran & Ghahari et al. 2011d; Ghahari et al. 2010 \\
\hline
\end{tabular}


Table 1 (continued). Updated list of Cotesia Cameron, 1891 species occurring in Iran.

\begin{tabular}{lll}
\hline Species of Cotesia & Distribution in Iran (Province) & References \\
\hline C. specularis (Szépligeti, 1896) & Qazvin & Ghahari et al. 2011c \\
C. spuria (Wesmael, 1837) & Qazvin & Ghahari et al. 2011c \\
C. telengai (Tobias, 1972) & Ilam & Ghahari et al. 2011b \\
C. tenebrosa (Wesmael, 1837) & Lorestan & Ghahari et al. 2012a \\
C. tibialis (Curtis, 1830) & Golestan & Ghahari et al. 2011b \\
C. vanessae (Reinhard, 1880) & Sistan \& Baluchestan, & Khajeh et al. 2014; \\
& West Azarbaijan & Karimpour et al. 2001 \\
C. vestalis (Haliday, 1834) & Alborz, East Azarbaijan, & Golizadeh et al. 2007; Rastegar et al. 2012; \\
& Isfahan, Khuzestan, Tehran & Ghahari et al. 2011a; present study; \\
C. villana (Reinhard, 1880) & & Hassanshahi et al. 2012 \\
C. zagrosensisZargar \& Gupta sp. nov. & Khuzestan & Ghahari et al. 2011c \\
C. zygaenarum (Marshall, 1885) & Golestan, Ilam, Mazandaran & Samin et al. 2015; Ghahari et al. 2011b; \\
\hline
\end{tabular}

Plutellidae Guenee, 1845, (Long \& Belokobylskij 2003; Yu et al. 2016), Pterophoridae Zeller, 1841, Pyralidae Latreille, 1803 (Shenefelt 1972), Tortricidae Latreille, 1803 (Yu et al. 2016).

\section{Discussion}

In total, nine species of the genus Cotesia are identified, of which three are new and described and illustrated here: C. elongata sp. nov., C. khuzestanensis sp. nov. and C. zagrosensis sp. nov. Moreover, C. cynthiae (Nixon, 1974) and Cotesia glabrata (Telenga, 1955) are recorded for the first time from Iran. Of all the species identified, Cotesia vestalis (Haliday, 1834) and C. elongata sp. nov. are the most predominant species, with almost $90 \%$ of all specimens collected in different parts of Khuzestan Province.

Of the three newly described species, C. khuzestanensis sp. nov. and C. elongata sp. nov. belong to the tibialis subgroup based on the following distinctive characters: outer side of metacoxa evenly rugose (in C. elongata sp. nov.) and punctuate-rugulose (in C. khuzestanensis sp. nov.). Cotesia khuzestanensis sp. nov., when compared with the congeneric Palearctic $C$. setebis, can be easily separated by the predominant character: first tergite being parallel sided and about 1.3 times as long as its apical width; it can be separated from the other congenerics C. ordinaria (Ratzeburg, 1844) (distributed in the Palearctic and Oriental regions) and C. orestes (Nixon, 1974) (distributed in the Palearctic region) by the following combination of characters: inner spur of metatibia slightly longer than outer, as long as half of metabasitarsus; penultimate segment of antenna 1.4 times as long as wide and $r$ oblique to fore margin of pterostigma. Cotesia elongata can be separated from the closely allied species C. ruficrus by the penultimate segment of the antenna 2-2.2 (-2.3) times as long as wide and the third tergite $0.7-0.8$ times as long as the second tergite. Cotesia zagrosensis sp. nov. belongs to the glomeratus subgroup based on the outer side of the metacoxa being smooth with no evident sculpture. Cotesia zagrosensis sp. nov. can be easily separated from $C$. onaspis and $C$. notha by the predominant character: the second tergite is conspicuously transverse and four times as long as the median length.

The majority of the taxonomic or biological studies on the genus Cotesia include the central and northern parts of Iran (Davatchi \& Chodjai 1969; Modarres Awal 1997; Karimpour et al. 2001; Abbasipour \& 
Taghavi 2002, 2004; Alizadeh \& Moghaddam 2004; Ghahari et al. 2009, 2011a, 2011b, 2011c, 2011d, 2012a, 2012b; Lashkari Bod et al. 2011; Naderian et al. 2012; Ghahari \& Fischer 2012; Sakenin et al. 2012; Khajeh et al. 2014; Samin 2015; Farahani et al. 2016; Samin et al. 2018). Prior studies reported 34 species of Cotesia from Iran. With three new species and two new records in the present study, the total number of species is raised to 39 (Table 1). Additionally, with the description of three new species, the total number of species of Cotesia across the globe reaches approximately three hundred.

Despite the current species diversity of the genus in Iran now being 39 species, more species can be expected to be found in the future when more comprehensive collections and studies are done. Further integrated taxonomic, molecular and faunistic research along with the host association is essentially required for this genus to better understand its species complexity. When compared with other geographical regions of the world, the known diversity of Cotesia in Iran is still preliminary. Many new taxa are likely to be discovered from Iran in the future, and thus this checklist will need to be periodically updated.

\section{Acknowledgements}

MZ is thankful to Tarbiat Modares University, Iran and the Ministry of Science, Iran for providing funds to visit ICAR-NBAIR, Bengaluru, India. AG is grateful to the Department of Agriculture Research and Education (DARE), Government of India (F. No.4-37/2017-IC.III (Trg.) dated $13^{\text {th }}$ April 2018), ICAR-National Bureau of Agricultural Insect Resources and Dr. C.R. Ballal (Director, ICARNBAIR) for facilitating a visit of MZ to India. We cordially thank Dr. Gavin Broad (topical editor of the European Journal of Taxonomy) and the two reviewers, Dr. Jose Fernandez-Triana (CNC, Ottawa, Canada) and Dr. Erinn Fagan-Jeffries (University of Adelaide, Australia), for their valuable comments and recommendations, which significantly improved the manuscript.

\section{References}

Abbasipour H. \& Taghavi A. 2002. Parasitoids of rice armyworm, Mythimna unipunctata (Lepidoptera: Noctuidae) in the rice fields of western Mazandaran. In: Proceedings of $15^{\text {th }}$ Iranian Plant Protection Congress Vol. 1: 41. Razi University, Kermanshah.

Abbasipour H. \& Taghavi A. 2004. Introduction of the rice armyworm, Mythimna unipuncta (Haworth) (Leoidoptera: Noctuidae) parasitoids in western Mazandaran rice fields and preliminary study on their efficiency to control the pest. Journal of Agricultural Science 1 (1): 19-28.

Abdoli P., Talebi A.A., Farahani S. \& Fernandez-Triana J. 2019a. Three new species of the genus Choeras Mason, 1981 (Hymenoptera: Braconidae, Microgastrinae) from Iran. Zootaxa 4545 (1): 77-92. https://doi.org/10.11646/zootaxa.4545.1.4

Abdoli P., Talebi A.A. \& Farahani S. 2019b. Dolichogenidea fernandeztrianai sp. nov. (Hymenoptera: Braconidae, Microgastrinae) from Iran. Journal of Agricultural Science and Technology 21 (3): 647658. Available from http://journals.modares.ac.ir/article-23-26466-en.html [accessed 16 Sep. 2019].

Alizadeh S. \& Moghaddam H. 2004. Introduction of some natural enemies of common cutworm (Agrotis segetum Schiff.) in Miyandoab. In: Proceeding of 16 $6^{\text {th }}$ Iranian Plant Protection Congress, Vol. 1: 45. University of Tabriz, Tabriz.

Balevski N.A. 1980. New species of Apanteles Foerst. (Hymenoptera, Braconidae) from Bulgaria. Entomological Review 59: 93-106.

Cameron P. 1891. Hymenopterological notices. I. On some Hymenoptera parasitic in Indian injurious insects. Memoirs and Proceedings of the Manchester Literary and Philosophical Society 4: 182-194. 
Capek M. 1972. Verzeichnis der aus Schädlingsinsekten erzogenen Parasiten. Teil IV Die Brackwespen (Braconidae, Hymenoptera). Entomological Problémy 10: 125-140.

Davatchi A. \& Chodjai M. 1969. Les hymenoptères entomophages de l'Iran (Etudes faunistiques). Université de Teheran, Faculté d'Agronomia 107: 1-88.

Farahani S., Talebi A.A., van Achterberg C. \& Rakhshani E. 2014. First record of Microplitis rufiventris Kokujev, 1914 (Braconidae: Microgastrinae) from Iran. Check List 10 (2): 441-444. https://doi.org/10.15560/10.2.441

Farahani S., Talebi A.A. \& Rakhshani E. 2016. Iranian Braconidae (Insecta: Hymenoptera: Ichneumonoidea): diversity, distribution and host association. Journal of Insect Biodiversity and Systematics 2 (1): 1-92.

Available from http://journals.modares.ac.ir/article-36-1141-en.html [accessed 16 Sep. 2019].

Fernández-Triana J.L., Whitfield J.B., Rodriguez J.J., Smith M.A., Janzen D.H., Hallwachs W., Hajibabaei M., Burns J.M., Solis M.A., Brown J., Cardinal S., Goulet H. \& Hebert P.D.N. 2014. Review of Apanteles sensu stricto (Hymenoptera: Braconidae, Microgastrinae) from Area de Conservación Guanacaste, northwestern Costa Rica, with keys to all described species from Mesoamerica. ZooKeys 383: 1-565. https://doi.org/10.3897/zookeys.383.6418

Gadallah N.S., Ghahari H. \& Peris-Felipo F.J. 2015. Catalogue of the Iranian Microgastrinae (Hymenoptera: Braconidae). Zootaxa 4043 (1): 1-69. https://doi.org/10.11646/zootaxa.4043.1.1

Ghafouri Moghaddam M., Rakhshani E., van Achterberg C. \& Mokhtari A. 2018. A study of the Iranian species of Choeras Mason (Hymenoptera: Braconidae: Microgastrinae), with the description of a new species. Zootaxa 4446 (4): 455-476. https://doi.org/10.11646/zootaxa.4446.4.3

Ghahari H. \& Fischer M. 2012. A faunistic survey on the braconid wasps (Hymenoptera: Braconidae) from Kermanshah province, Iran. Entomofauna 33 (20): 305-312.

Ghahari H., Tabari M., Haji-Amiri M., Sakenin H. \& Ostovan H. 2009. Population fluctuation of rice stem borer, Chilo suppressalis Walker (Lepidoptera: Pyralidae) in paddy fields of northern Amol in Mazandaran Province. Journal of Plant Protection 23 (1): 41-49. [In Persian, with English abstract.]

Ghahari H., Cetin Erdoğan O., Šedivý J. \& Ostovan H. 2010. Survey of the Ichneumonoidea and Chalcidoidea (Hymenoptera) parasitoids of Saturniidae (Lepidoptera) in Iran. Entomofauna 10: 1-6.

Ghahari H., Fischer M. \& Papp J. 2011a. A study on the braconid wasps (Hymenoptera: Braconidae) from Isfahan Province, Iran. Entomofauna 32 (16): 261-272.

Ghahari H., Fischer M. \& Papp J. 2011b. A study on the Braconidae (Hymenoptera: Ichneumonoidea) from Ilam Province, Iran. Calodema 160: 1-5.

Ghahari H., Fischer M. \& Papp J. 2011c. A study on the Braconidae (Hymenoptera: Ichneumonoidea) from Qazvin province, Iran. Entomofauna 32 (9): 197-208.

Ghahari H., Fischer M., Sakenin H. \& Imani S. 2011d. A contribution to the Agathidinae, Alysiinae, Aphidiinae, Braconinae, Microgastrinae and Opiinae (Hymenoptera: Braconidae) from cotton fields and surrounding grasslands of Iran. Linzer biologische Beiträge 43 (2): 1269-1276.

Ghahari H., Fischer M., Papp J. \& Tobias V. 2012a. A contribution to the knowledge of braconids (Hymenoptera: Braconidae) from Lorestan province Iran. Entomofauna 33 (7): 65-72.

Ghahari H., Fischer M. \& Tobias V. 2012b. A study on the Braconidae (Hymenoptera: Ichneumonoidea) from Guilan province, Iran. Entomofauna 33 (22): 317-324.

Golizadeh A., Kamali K., Fathipour Y., Abbasipour H. \& Lozan A. 2007. Report of the parasitoid wasp, Cotesiaplutellae (Hym.: Braconidae), from Iran. Journal of Entomological Society of Iran 27 (2): 19-20. 
Gupta A. \& Fernández-Triana J.L. 2014. Diversity, host association, and cocoon variability of reared Indian Microgastrinae (Hymenoptera: Braconidae) Zootaxa 3800 (1): 1-101.

https://doi.org/10.11646/zootaxa.3800.1.1

Gupta A., Venkatesan T. \& More R.P. 2016a. Morphological and molecular characterization of reared parasitoid wasps of the genus Glyptapanteles Ashmead 1904 (Insecta: Hymenoptera: Braconidae: Microgastrinae) associated with Lepidoptera in India. PLoS ONE 11 (3): e0150765.

https://doi.org/10.1371/journal.pone.0150765

Gupta A., Shaw M., Cardinal S. \& Fernandez-Triana J.L. 2016b. A review of unusual species of Cotesia (Hymenoptera, Braconidae, Microgastrinae) with the first tergite narrowing at midlength. ZooKeys 580: 29-44. https://doi.org/10.3897/zookeys.580.8090

Haliday A.H. 1834. Essay on parasitic Hymenoptera. Entomological Magazine 2 (3): 225-259.

Hassanshahi G., Askarianzadeh A., Abbasipour H. \& Karimi J. 2012. Natural parasitism of diamondback moth, Plutella xylostella L. (Lep.: Plutellidae) on different cultivars of cauliflower. In: Proceedings of $20^{\text {th }}$ Iranian Plant Protection Congress, Vol. 1: 11. University of Shiraz, Shiraz.

Karimpour Y., Fathipour Y., Talebi A.A. \& Moharramipour S. 2001. Report of two endoparasitoid wasps, Cotesia ofella (Nixon) and Cotesia vanessae (Hym.: Braconidae) on larvae of Simara dentinosa Freyer (Lep.: Noctuidae) from Iran. Journal of Entomological Society of Iran 21 (2): 106.

Khajeh N., Rakhshani E., Arjmandi A.A. \& Barahoei H. 2014. A faunistic study on Microgastrinae in Sistan region. In: Proceedings of $21^{\text {th }}$ Iranian Plant Protection Congress, Vol. 1: 575. University of Orumiyeh, Orumiyeh.

Khanjani M. 2006. Vegetable Pests in Iran. Bu-Ali Sina University Press, Hamedan. [In Persian.]

Kurdjumov N.V. 1912. Hyménoptères-parasites nouveaux ou peu connus. Revue russe d'Entomologie 12: 223-240.

Lashkari Bod A., Rakhshani E., Talebi A.A., Lozan A. \& Zikic V. 2011. A contribution to the knowledge of Braconidae (Hym., Ichneumonoidea) of Iran. Biharean Biologist 5 (2): 147-150.

Long K.D. \& Belokobylskij S. 2003. A preliminary list of the Braconidae (Hymenoptera) of Vietnam. Russian Entomological Journal 12 (4): 385-398.

Marshall T. A. 1885. Monograph of the British Braconidae, I. Transactions of the Entomological Society, London.

Mason W.R.M. 1981. The polyphyletic nature of Apanteles Förster (Hymenoptera: Braconidae): a phylogeny and reclassification of Microgastrinae. Memoirs of the Entomological Society of Canada 115: 1-147. https://doi.org/10.4039/entm113115fv

Michel-Salzat A. \& Whitfield J.B. 2004. Phylogenetic relationships among Microgastrinae braconid wasps genera based on data from the 16S, COI and 28S genes and morphology. Systematic Entomology 27: 337-359. https://doi.org/10.1046/j.1365-3113.2002.00183.x

Modarres Awal M. 1997. Family Braconidae (Hymenoptera). In: Modarres Awal M. (ed.) List of Agricultural Pests and their Natural Enemies in Iran: 265-267. Ferdowsi University Press, Mashhad.

Naderian H., Ghahari H. \& Asgari S. 2012. Species diversity of natural enemies in corn fields and surrounding grasslands of Semnan province, Iran. Calodema 217: 1-8.

Nixon G.E.J. 1965. A reclassification of the tribe Microgasterini (Hymenoptera: Braconidae). Bulletin of the British Museum (Natural History) Entomology, Supplement 2: 1-284. 
Nixon G.E.J. 1974. A revision of the north-western European species of the glomeratus-group of Apanteles Foerster (Hymenoptera, Braconidae). Bulletin of Entomological Research 64: 453-524.

Papp J. 1986. A survey of the European species of Apanteles Först. (Hymenoptera, Braconidae: Microgastrinae). IX. The glomeratus-group, 1. Annales Historico-Naturales Musei Nationalis Hungarici 78: 225-247.

Papp J. 1987. A survey of the European species of Apanteles Först. (Hymenoptera, Braconidae: Microgastrinae). X. The glomeratus-group, 2 and the cultellatus-group. Annales Historico-Naturales Musei Nationalis Hungarici 79: 207-258.

Quicke D.L.J. 2015. The Braconid and Ichneumonid Parasitoid Wasps: Biology, Systematics, Evolution and Ecology. Willey-Blackwell, Oxford.

Rassipour A. 1983. Étude biologique d'Apanteles chilonis Mun. (Hym.: Braconidae) en vue de la lutte biologique contre la pyrale du riz, Chilo suppressalis Walk. (Lep.: Pyralidae). Bulletin Plant Protection Organization, Iran 29: 1-24.

Rastegar J., Sakenin H., Khodaparast S. \& Havaskary M. 2012. On a collection of Braconidae (Hymenoptera) from East Azarbaijan and vicinity, Iran. Calodema 226: 1-4.

Razmi M., Karimpour Y., Safaralizadeh M.H. \& Safavi S.A. 2011. Parasitoid complex of cabbage large white butterfly Pierisbrassicae (L.) (Lepidoptera: Pieridae) in Urmia with new records from Iran. Journal of Plant Protection Research 51 (3): 248-251.

Rodriguez J.J., Fernandez-Triana J.L., Smith M.A., Janzen D.H., Hallwachs W., Erwin T. L. \& Whitfield J.B. 2013. Extrapolations from field studies and known faunas converge on dramatically increased estimates of global Microgastrine parasitoid wasp species richness (Hymenoptera: Braconidae). Insect Conservation and Diversity 6 (4): 530-536. https://doi.org/10.1111/icad.12003

Rousse P. \& Gupta A. 2013. Microgastrinae (Hymenoptera: Braconidae) of Réunion Island: a catalogue of the local species. Zootaxa 3616 (6): 501-547. https://doi.org/10.11646/zootaxa.3616.6.1

Sakenin H., Naderian H., Samin N., Rastegar J., Tabari M. \& Papp J. 2012. On a collection of Braconidae (Hymenoptera) from northern Iran. Linzer biologische Beiträge 44 (2): 1319-1330.

Samin N. 2015. A faunistic study on the Braconidae of Iran (Hymenoptera: Ichneumonoidea). Arquivos Entomoloxicos 13: 339-345.

Samin N., Coronado-Blanco J.M., Fischer M., van Achterberg K., Sakenin H. \& Davidian E. 2018. Updated checklist of Iranian Braconidae (Hymenoptera: Ichneumonoidea) with twenty-three new records. Natura Somogyiensis 32: 21-36.

Shaw M.R. 2012. Larval parasitoids of Rivula sericealis (Scopoli) (Lepidoptera: Noctuidae) in Britain, including notes on the biology of Cotesia subordinaria (Tobias) (Hymenoptera: Braconidae, Microgastrinae), a solitarycum-gregarious parasitoid. Entomologist's Gazette 63: 251-7.

Shenefelt R.D. 1972. Braconidae 4. Microgastrinae Apanteles Foerster. Hymenopterorum Catalogus 4: 429-668.

Szépligeti G. 1904. Hymenoptera. Fam. Braconidae. Genera Insectorum 22: 1-253.

Telenga N.A. 1955. Braconidae, subfamily Microgasterinae, subfamily Agathinae. Fauna USSR, Hymenoptera 5: 1-311.

Thomson C. G. 1895. LVII Bidrag till braconidernas kännedom. Opuscula Entomologica 20: 21412339. Available from https://biodiversitylibrary.org/page/9666252 [accessed 18 Oct. 2019]. 
Tobias V.I. 1986. Subfamily Braconinae. In: Medvedev G.S. (ed.) Keys to the Insects of the European Part of the USSR. Vol.3. Hymenoptera. Part 4: 156-254. Nauka Publisher, Leningrad.

Van Achterberg C. 1993. Illustrated key to the subfamilies of the Braconidae (Hymenoptera: Ichneumonoidea). Zoologische Verhandelingen Leiden 283: 1-189.

Van Achterberg C. \& Polaszek A. 1996. The parasites of cereal stem borers (Lepidoptera: Cossidae, Crambidae, Noctuidae, Pyralidae) in Africa, belonging to the family Braconidae (Hymenoptera: Ichneumonoidea). Zoologische Verhandelingen 304: 1-123.

Yu D.S.K., van Achterberg C. \& Horstmann K. 2016. Taxapad 2016, Ichneumonoidea 2015. Database on flash-drive, Nepean, Ontario, Canada.

Zargar M., Gupta A., Talebi A.A. \& Farahani S. 2019a. A review of the Iranian species of genus Iconella Mason (Hymenoptera: Braconidae: Microgastrinae) with description of two new species. Zootaxa 4586 (3): 491-504. https://doi.org/10.11646/zootaxa.4586.3.6

Zargar M., Gupta A., Talebi A.A. \& Farahani S. 2019b. Description of a new species of the genus Deuterixys (Hymenoptera: Braconidae: Microgastrinae) from Iran. Biologia (Accepted).

https://doi.org/10.2478/s11756-019-00298-9

Manuscript received: 8 May 2019

Manuscript accepted: 15 July 2019

Published on: 24 October 2019

Topic editor: Gavin Broad

Desk editor: Kristiaan Hoedemakers

Printed versions of all papers are also deposited in the libraries of the institutes that are members of the EJT consortium: Muséum national d'histoire naturelle, Paris, France; Meise Botanic Garden, Belgium; Royal Museum for Central Africa, Tervuren, Belgium; Royal Belgian Institute of Natural Sciences, Brussels, Belgium; Natural History Museum of Denmark, Copenhagen, Denmark; Naturalis Biodiversity Center, Leiden, the Netherlands; Museo Nacional de Ciencias Naturales-CSIC, Madrid, Spain; Real Jardín Botánico de Madrid CSIC, Spain; Zoological Research Museum Alexander Koenig, Bonn, Germany; National Museum, Prague, Czech Republic. 